ARTICLE

\title{
LIGHT/LT $\beta R$ signaling regulates self-renewal and differentiation of hematopoietic and leukemia stem cells
}

\author{
S. S. Höpner 1,2, Ana Raykova1,2, R. Radpour (10 1,2, M. A. Amrein (1) 1,2, D. Koller,2, G. M. Baerlocher ${ }^{3}$, \\ C. Riether (D) ${ }^{1,2} \&$ A. F. Ochsenbein (D) ${ }^{1,2} \otimes$
}

The production of blood cells during steady-state and increased demand depends on the regulation of hematopoietic stem cell (HSC) self-renewal and differentiation. Similarly, the balance between self-renewal and differentiation of leukemia stem cells (LSCs) is crucial in the pathogenesis of leukemia. Here, we document that the TNF receptor superfamily member lymphotoxin- $\beta$ receptor (LT $\beta R$ ) and its ligand LIGHT regulate quiescence and self-renewal of murine and human HSCs and LSCs. Cell-autonomous LIGHT/LTßR signaling on HSCs reduces cell cycling, promotes symmetric cell division and prevents primitive HSCs from exhaustion in serial re-transplantation experiments and genotoxic stress. LT $\beta R$ deficiency reduces the numbers of LSCs and prolongs survival in a murine chronic myeloid leukemia (CML) model. Similarly, LIGHT/LT $\beta R$ signaling in human G-CSF mobilized HSCs and human LSCs results in increased colony forming capacity in vitro. Thus, our results define LIGHT/ LT $\beta R$ signaling as an important pathway in the regulation of the self-renewal of HSCs and LSCs.

\footnotetext{
${ }^{1}$ Department of Medical Oncology, Inselspital, Bern University Hospital, University of Bern, Bern, Switzerland. ${ }^{2}$ Department for BioMedical Research, University of Bern, Bern, Switzerland. ${ }^{3}$ Department of Hematology and Central Hematology Laboratory, Inselspital, Bern University Hospital, University of Bern, Bern, Switzerland. ${ }_{\text {email: adrian.ochsenbein@insel.ch }}$
} 
ematopoietic stem cells (HSCs) represent a small heterogeneous and hierarchically organized population within lineage negative $\left(\mathrm{Lin}^{-}\right)$cells ${ }^{1}$. The hierarchy of HSCs is characterized by the progressive decrease of the selfrenewal capability from long-term-HSCs (LT-HSCs) over shortterm HSCs (ST-HSCs) to multipotent progenitors (MPPs). HSC self-renewal and differentiation is regulated by cell-intrinsic mechanisms such as transcription factors (SATB1, PU-1), cell cycle regulators (CDKN1A, GFI-1), or transcriptional regulators $(\mathrm{MSI} 2)^{2-4}$. In addition, cell-extrinsic cues from bone marrow (BM) niche cells regulate HSC activity, that is, niche cells secrete stem cell factor (SCF), angiopoietin-1, and thrombopoietin ${ }^{5}$, and express adhesion molecules such as vascular cell adhesion protein 1 (ref. $\left.{ }^{6}\right)$. In addition, stromal cell-derived factor 1 (CXCL12) promotes the retention of HSCs in the BM and contributes to quiescence ${ }^{7}$.

Importantly, HSCs are able to adapt rapidly to increased demands triggered by infections, inflammation, chemotherapeutic agents, or ionizing radiation with increased cell cycling and the production of hematopoietic progenitors ${ }^{8}$. This demandadapted hematopoiesis is activated by immune effector cytokines such as interferon- $\alpha$, interferon- $\gamma$, and tumor necrosis factor- $\alpha$ (TNF- $\alpha$ ) and Toll-like receptors (TLRs) that sense conserved microbial products ${ }^{9,10}$

To ensure lifelong production of all hematopoietic lineages, not only the cell division of HSC but also the cell division fate, for example, differentiation, has to be tightly controlled ${ }^{11}$. Asymmetric cell division, leading to a differentiated and a selfreplicable daughter cell, assures a constant stem cell pool and a progressive increase of differentiated cells. Symmetric division leads to two identical daughter cells and the expansion of the stem cell pool or, in rare situations, to two identical more differentiated cells ${ }^{12}$.

Leukemic stem cells (LSCs) are responsible for leukemia initiation and propagation ${ }^{13}$. They share several characteristics with HSCs, including quiescence, self-renewal, and the capacity to undergo symmetric and asymmetric cell division. Importantly, most of the molecular pathways regulating HSCs also control LSCs $^{14,15}$ In addition, we and others showed that the TNF receptor (TNFR) family member CD27 regulates self-renewal and differentiation of HSCs and LSCs ${ }^{16-19}$.

The lymphotoxin- $\beta$ receptor (LT $\beta$ R), another member of the TNFR family, is expressed on epithelial, stromal, and myeloid cells but not on lymphocytes. Lymphotoxins are cytokines that guide the interaction of immune cells, mainly lymphocytes, with the surrounding stromal cells ${ }^{20}$. Lymphotoxin- $\alpha$ (LTa) and LT $\beta$ as heterotrimers (LTa1 $\beta 2$ ) and LIGHT are known ligands for $L T \beta R^{21}$. LT $\beta R$ signaling is crucially involved in the development and organization of lymphoid tissue $\mathrm{e}^{21}$. Therefore, secondary lymphoid tissue is absent in $L t a, L t b$, and $L t b r$-knockout mice but, interestingly, not in Light-knockout mice ${ }^{22,23}$. Ligation of LT $\beta R$ leads to TNFR-associated factor 2 (TRAF2)-NIK-mediated activation of the classical and alternative nuclear factor $-\kappa \mathrm{B}(\mathrm{NF}-\kappa \mathrm{B})$ pathway and the transcription of genes involved in inflammation and development ${ }^{24}$. Disruption of LT $\beta \mathrm{R}$ signaling results in impaired protection to viral and bacterial infections ${ }^{25,26}$. In addition, cancer cells may express LT $\beta \mathrm{R}$ and agonistic antibodies to LT $\beta \mathrm{R}$ trigger cancer cell death and suppress tumor growth in vivo $^{27}$.

In this work, we document that LIGHT expressed by HSCs induces quiescence and self-renewal by cell-autonomous ligation of LT $\beta$ R. Importantly, LIGHT expression is upregulated in HSCs in response to increased demands of progenitors and differentiated blood cells. LIGHT/LT $\beta$ R signaling reduces cell cycling of HSCs, promotes symmetric over asymmetric cell division, and, thereby, maintains the pool of primitive HSCs. Although the maintenance of primitive HSC is crucial to secure hematopoiesis long-term, the accumulation of primitive LSCs and the lack of differentiation promote disease progression in leukemia ${ }^{28}$. Indeed, LIGHT/LT $\beta R$ signaling maintains and expands the pool of LSCs in a murine model of chronic myeloid leukemia (CML) and promotes disease progression. Similarly, LIGHT/LT $\beta$ R signaling induces stemness in human CML stem/progenitor cells. Importantly, LIGHT is upregulated in LSCs compared to normal HSCs. This may allow selectively targeting LSCs and inducing differentiation. Collectively, this study identifies the LIGHT/LT $\beta$ R pathway as a crucial regulator of self-renewal of HSCs and LSCs.

\section{Results}

LT $\beta$ R regulates self-renewal of HSCs. First, we analyzed the expression of LT $\beta \mathrm{R}$ on different murine hematopoietic stem/progenitor cell (HSPC) subsets during homeostasis. ImageStreamX MkII analysis of fluorescence-activated cell sorting (FACS)-purified $\mathrm{Lin}^{-} \mathrm{scal}^{+} \mathrm{c}$-kit ${ }^{+}$cells (LSKs) from BM revealed a strong surface expression of LT $\beta$ R (Fig. 1a). In addition, defined LSK subpopulations such as LT-HSCs $\left(\mathrm{CD} 150^{+} \mathrm{CD} 48^{-}\right.$ $\left.\mathrm{CD}^{-}{ }^{-} \mathrm{CD} 135^{-}\right)$, ST-HSCs $\left(\mathrm{CD} 150^{+} \mathrm{CD} 48^{-} \mathrm{CD}^{-} 4^{+} \mathrm{CD} 135^{-}\right)$, MPPs (MPP1: $\mathrm{CD} 150^{+} \mathrm{CD} 48^{+} \mathrm{CD} 34^{+} \mathrm{CD} 135^{-}$; $\mathrm{MPP} 2: \mathrm{CD}^{2} 50^{-}$ $\mathrm{CD}^{+}{ }^{+} \mathrm{CD} 34^{+} \mathrm{CD} 135^{-}$; $\left.\mathrm{MPP} 3: \mathrm{CD}_{150}{ }^{-} \mathrm{CD} 48^{+} \mathrm{CD} 34^{+} \mathrm{CD} 135^{+}\right)$, as well as common myeloid progenitors $\left(\mathrm{CD} 34^{+} \mathrm{Fc} \gamma \mathrm{RII} / \mathrm{III}^{-} \mathrm{c}-\mathrm{kit}^{+}\right.$

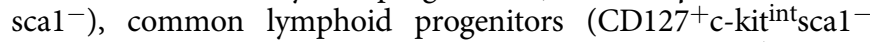
Thy $\left.1.1^{-}\right)$, granulocyte-macrophage progenitors (CD34+ ${ }^{+} \mathrm{F} \gamma \mathrm{RII} /$ $\left.\mathrm{III}^{+} \mathrm{c}-\mathrm{kit}^{+} \mathrm{scal}^{-}\right)$, and megakaryocyte-erythroid progenitors

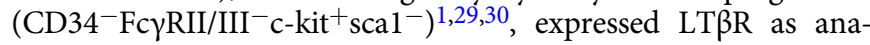
lyzed by FACS (Fig. 1b and Supplementary Fig. 1a). Furthermore, differentiated myeloid cells in the BM and peripheral blood (PB) expressed LT $\beta$ R, whereas T- and B-lymphocytes only marginally expressed LT $\beta$ R (Supplementary Fig. 1b).

To analyze the functional relevance of LT $\beta R$ expressed on hematopoietic cells, we first compared hematopoiesis in naive $\mathrm{BL} / 6$ and $\mathrm{Ltbr}^{-1-}$ mice. BL/6 and $\mathrm{Ltbr}^{-1-}$ mice had similar numbers of LSKs (LT-HSC, ST-HSC, and MPPs), lymphoid progenitors (common lymphoid progenitors), and myeloid progenitors (granulocyte-macrophage progenitors, common myeloid progenitors, megakaryocyte-erythroid progenitors) in the BM (Fig. 1c-f). In addition, BL/6 and $\mathrm{Ltbr}^{-1-}$ LSKs had a comparable cell cycle activity and apoptosis rate (Supplementary Fig. 1c, d). Moreover, FACS-purified LSKs from BL/6 and $\mathrm{Ltbr}^{-1-}$ mice formed similar numbers of colonies in methylcellulose ex vivo (Fig. $1 \mathrm{~g}$ ). Since not only HSCs but also early progenitor cells form colonies ex vivo in methylcellulose ${ }^{31,32}$, we analyzed the self-renewal capacity of HSCs in serial re-plating experiments ${ }^{33}$. Ltbr ${ }^{-/}$LSKs lost the capacity to form colonies in methylcellulose after the third and fourth replating, indicating that LT $\beta \mathrm{R}$ signaling contributes to the selfrenewal of HSCs (Fig. 1g).

Since differences between BL/6 and $\mathrm{Ltbr}^{-/}$- HSCs only became apparent in serial re-plating assays in vitro that require cell expansion and differentiation, we determined the function of LT $\beta R$ on LSKs in vivo in a competitive repopulation experiment (Fig. 1h). We first injected congenic Ltbr-proficient (Ly5.1 ${ }^{+}$, referred to as Ly5.1) LSKs and LTßR-deficient (Ly5.2+ ${ }^{+}$, referred to as $\mathrm{Ltbr}^{-1-}$ ) LSKs into lethally irradiated Ly5.1/Ly5.2 recipient mice. Importantly, injected LSKs homed to the BM independent of LT $\beta$ R expression (Supplementary Fig. 1e). However, $\mathrm{Ltbr}^{-/-}$ LSKs reconstituted recipient mice more efficiently than control LSKs, resulting in $>60 \% \mathrm{Ltbr}^{-/-} \mathrm{Lin}^{-}$cells in the BM 16 weeks post transplantation (Fig. 1i and Supplementary Fig. 1f). This was also reflected by a significant higher fraction of all HSPC subpopulations (Fig. $1 \mathrm{j}-\mathrm{n}$ and Supplementary Fig. 1g-j) in the $\mathrm{BM}$ and higher numbers of $L t b r^{-I-}$ leukocytes in PB (Supplementary Fig. 1k). 


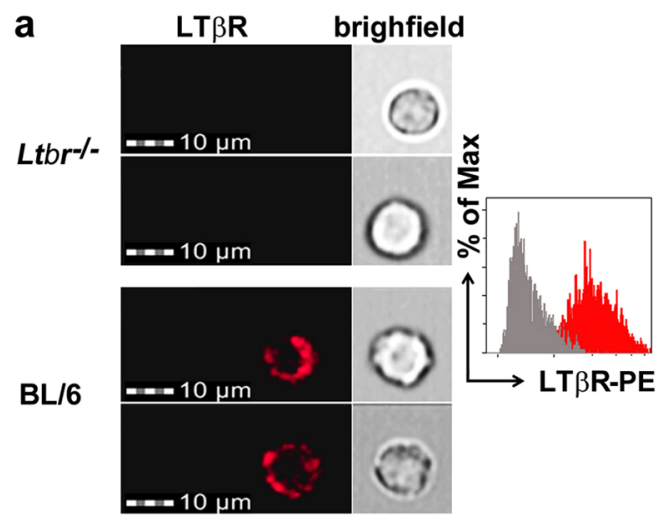

b LT-HSC
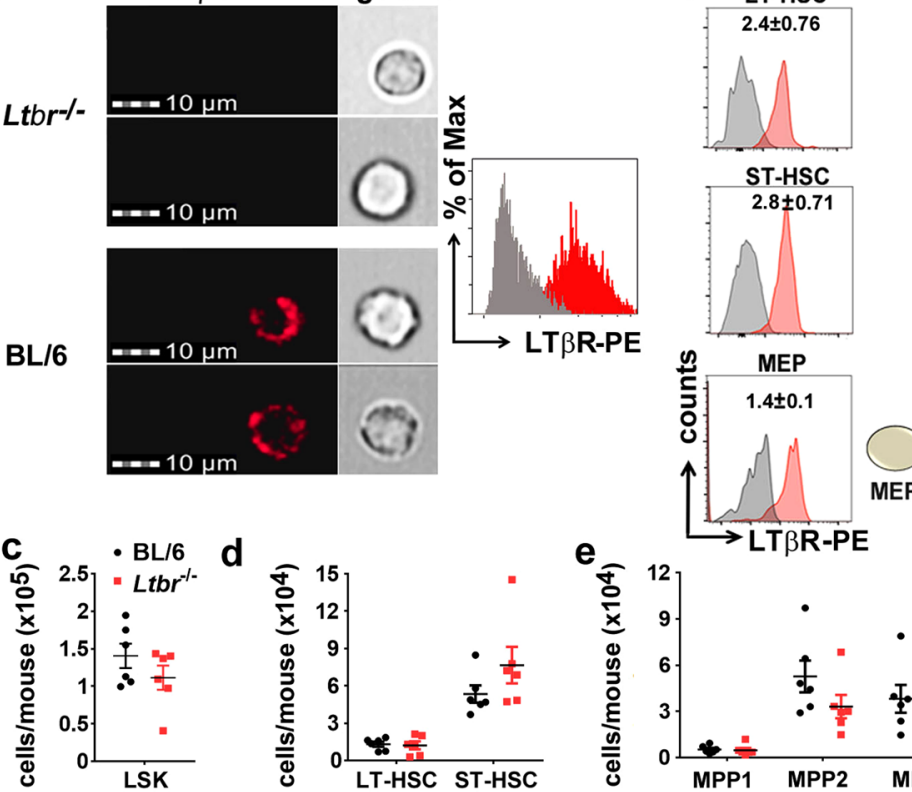

d
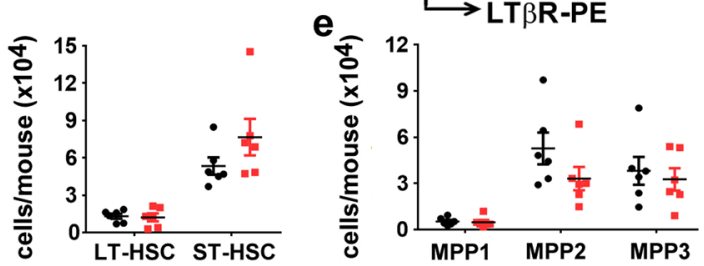

h
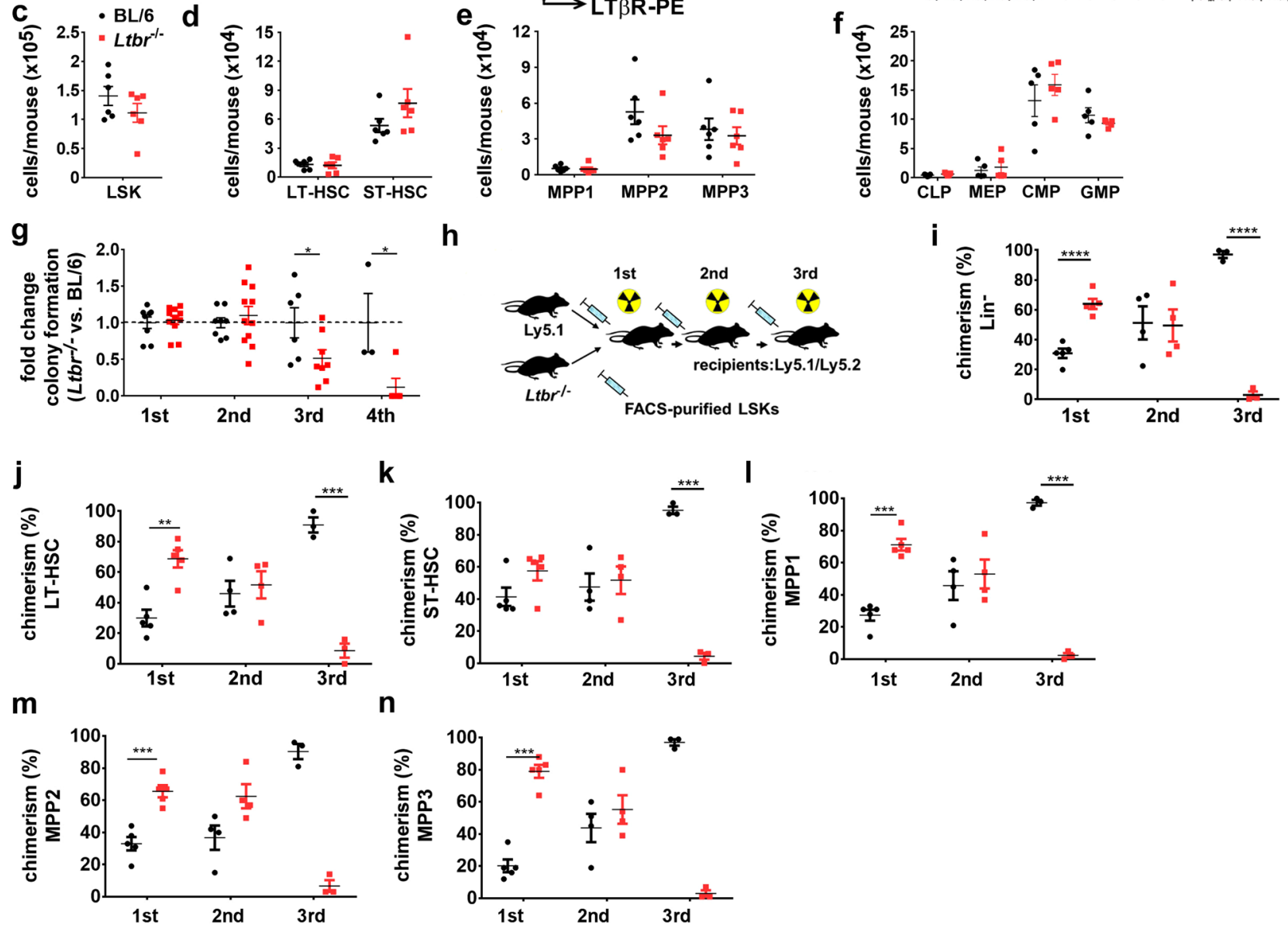

n

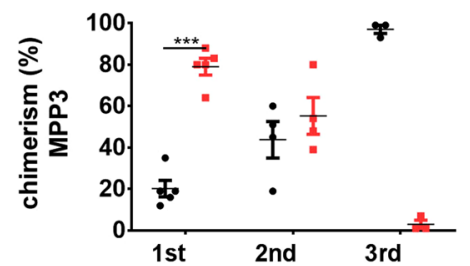

Fig. 1 LT $\beta$ R expressed by LSKs prevents exhaustion of HSCs. a Left: LT $\beta R$ expression (red) on BL/6 and Ltbr-/- FACS-purified BM LSKs analyzed by ImageStreamX MkII. Right: ImageStreamX Mkll histogram of LT $\beta R$ expression on Ltbr ${ }^{-/}$- LSKs (gray) vs. BL/6 LSKs (red). b Representative FACS histograms of LTRR expression on BM LSK subsets and myeloid progenitor cells. Numbers in plots indicate the ratio MFI stain/isotype ( $n=5$ mice). c-f Absolute numbers of LSKs (c), LT/ST-HSCs (d), MPPs (e), CLPs and myeloid progenitors (MEP, CMP, GMP) (f) in the BM of naive BL/6 (black, $n=6$ ) and $\mathrm{Ltbr}^{-/-}\left(\right.$red, $n=6$ ) mice. $\mathbf{g}$ Fold change in CFU of $L t b r^{-/}$LSKs relative to BL/6 LSKs in serial re-platings in vitro (1st: $n=8$ for BL/6 and 11 for $L_{t b r}{ }^{-/-} ; 2$ nd: $n=8$ for BL/ 6 and 11 for $L t b r^{-/-} ; 3 r d: n=6$ for BL/ 6 and 8 for $L t b r^{-/-} ; 4$ th: $n=3$ for BL/6 and 5 for $L$ tbr ${ }^{-/-}$, one out of two independent experiments). $\mathbf{h}$ Schematic of mixed BM chimera and serial re-transplantation. i-n Percentages of Ly5.1 and Ltbr ${ }^{-/}-$Lin $^{-}$cells (i) and LSK subsets (j-n), $n=5$ (1st), $n=4$ (2nd), $n=3$ (3rd), one out of two independent experiments is shown. Data are shown as mean \pm SEM. Statistics: ${ }^{\star} P<0.05$, ${ }^{\star \star} P<0.01$, ${ }^{\star \star \star} P<0.001,{ }^{\star \star \star \star} P<0.0001$ (two-tailed $t$ test); g: 1st $p=0.05,2$ nd $p=0.039 ; \mathbf{i}: 1$ st $p<0.0001,3 r d p<0.0001 ; \mathbf{j}: 1$ st $p=0.0012,3 r d p=0.0003 ; \mathbf{k}: 3 r d$ $p<0.0001$; I: 1st $p<0.0001$, 3rd $p<0.0001 ; \mathbf{m}: 1$ st $p=0.0004$, 3rd $p=0.0001 ; \mathbf{n}:$ st $p<0.0001$, 3rd $p<0.0001$.

To study the self-renewal capacity of HSCs, we performed serial re-transplantations with equal numbers of FACS-purified $\mathrm{Ltbr}^{-1-}$ and Ly5.1 LSKs. This resulted in a comparable reconstitution of HSPCs in the second transplantation (Fig. 1i-n and Supplementary Fig. 1f-j) and similar numbers of LT $\beta R$-competent and
LTßR-deficient leukocytes in blood (Supplementary Fig. 11). Importantly, $\mathrm{Ltbr}^{-1-}$ LSKs lost the capacity to reconstitute hematopoiesis in the third transplantation, resulting in only $\sim 3 \% \mathrm{Ltbr}^{-1-}$ HSPCs in BM and differentiated leukocytes in blood (Fig. 1i-n and Supplementary Fig. 1f-j, m). Taken together, these 
data indicate that LT $\beta$ R signaling crucially contributes to selfrenewal of HSCs.

Cell-autonomous expression of LIGHT induces LT $\beta$ R signaling and self-renewal of HSCs. LT $\beta R$ signaling can be induced by ligation with LTa1 $\beta 2$ heterotrimers and LIGHT ${ }^{34}$. Therefore, we analyzed messenger RNA (mRNA) expression of these ligands in naive Ly5.1 LSKs and BM niche cells, such as osteoblasts, endothelial cells (ECs), and mesenchymal stromal cells (MSCs). In addition, FACS-purified Ly5.1 LSKs from chimeras 6 weeks post transplantation were analyzed (Fig. 2a and Supplementary Fig. 2a). Lta and $L t b$ mRNA was expressed at very low levels in LSKs from both naive and chimeric mice. However, the membranous form of Light was expressed in naive LSKs and its expression was increased $~ 2.5$-fold in Ly5.1 LSKs isolated from chimeric mice (Fig. 2a). By contrast, LTRR ligands were only expressed at low levels in BM niche cells, with the exception of osteoblasts that showed higher Light expression (Supplementary Fig. 2a). Interestingly, Ltbr mRNA expression in LSKs from chimeric mice 6 weeks post transplantation was increased $\sim 12$ fold when compared to naive LSKs (Fig. 2b).

The high expression of LIGHT on LSKs prompted us to analyze its role in the maintenance and regulation of HSPCs. Similar to our results in $\mathrm{Ltbr}^{-1-}$ mice, the HSPC composition in the BM of naive BL/6 and Light ${ }^{-1-}$ mice was comparable (Supplementary Fig. 2b-d). In addition, no differences were found in cell cycle activity and apoptosis rate of LSKs in the absence of Light (Supplementary Fig. 2e, f). To further elucidate whether LIGHT-induced LT $\beta$ R signaling regulates LSK colony formation capacity in vitro, we performed a serial re-plating experiment. In line with our findings obtained with $\mathrm{Ltbr}^{-1-}$ LSKs, colony formation capacities of Light $^{-1-}$ LSKs were gradually lost in serial re-plating experiments in vitro (Fig. 2c).

These data suggest that LIGHT expressed by LSKs triggers LT $\beta R$ signaling and regulates colony formation in vitro. LT $\beta R$ expressed on LSKs may bind to LIGHT in trans (in which the ligand is expressed on another cell) or in cis (the ligand is expressed by the same cell as the receptor). To distinguish between cis- and trans-signaling, we performed mixed colony assays with FACS-purified green fluorescent protein (GFP)expressing LIGHT-proficient LSKs and LIGHT-deficient LSKs. LIGHT provided by surrounding LSKs did not rescue the colonyforming capacity of LIGHT-deficient LSKs in serial re-plating experiments (Fig. 2d). This indicates that cell-autonomous LIGHT/LT $\beta R$ signaling maintains HSC self-renewal capacity.

To study the self-renewal capacity of Light $^{-1-}$ LSKs in vivo, we performed competitive serial repopulation experiments (Fig. 2e). Light $^{-1-}$ LSKs reconstituted primary recipient mice more efficiently, resulting in a chimerism of $\sim 70 \%$ of $\mathrm{Lin}^{-} \mathrm{BM}$ cells and HSC subsets, as well as higher numbers of leukocytes in blood (Fig. 2f-j and Supplementary Fig. 2g-j). However, Light ${ }^{-1-}$ LSKs subsequently lost the capacity to reconstitute hematopoiesis in serial transplantations, resulting in $<10 \%$ Light $^{-1-}$ HSPCs and differentiated leukocytes in the fourth transplantation (Fig. $2 \mathrm{f}-\mathrm{j}$ and Supplementary Fig. $2 \mathrm{~g}-\mathrm{k}$ ). Similarly to the in vitro coculture experiments with LIGHT-proficient and LIGHT-deficient LSKs, LIGHT expressed by surrounding hematopoietic cells did not rescue the defect in self-renewal of LIGHT-deficient LSKs in vivo.

These experiments suggest that cell-autonomous (cis) LIGHT/ LT $\beta R$ signaling maintains LSK self-renewal. However, Light mRNA was also expressed on osteoblasts (Supplementary Fig. 2a). Therefore, we analyzed whether LIGHT-expressing cells of the $\mathrm{BM}$ microenvironment contribute to LT $\beta \mathrm{R}$ signaling in HSCs by transplanting Ly5.1 or Light ${ }^{-1-}\left(\right.$ Ly5.2 $\left.2^{+}\right)$LSKs into Ly5.1/Ly5.2 or Ly5.1/Light ${ }^{-l-}$ recipients (Fig. $2 \mathrm{k}$ ). This experiment revealed that LIGHT deficiency on host cells did not alter the reconstitution potential and self-renewal capacity of Ly5.1 HSPCs in serial re-transplantation experiments in vivo. By contrast, Light ${ }^{-1-}$ LSKs reconstituted hematopoiesis more efficiently in first transplantation and then gradually lost the capacity to reconstitute hematopoiesis in second, third, and fourth transplantations, independently of the expression of LIGHT in recipient mice (Fig. 2l-o and Supplementary Fig. 2l-o). This indicates that LIGHT ligates LT $\beta$ R in a cell-autonomous fashion in vivo leading to LT-HSC self-renewal.

Loss of LT $\beta R$ increases cell cycle-related gene expression while reducing the expression of genes associated with stemness. In order to study the molecular mechanisms of LT $\beta \mathrm{R}$-mediated HSC regulation, we performed a transcriptomic analysis of Ly5.1 and $\mathrm{Ltbr}^{-1-}$ LT/ST-HSCs from chimeric mice using RNA-sequencing (RNA-seq) analysis (Fig. 3a). Ly5.1 or $\mathrm{Ltbr}^{-1-}$ LT/ST-HSCs from different chimeras had similar gene expression profiles and therefore clustered together in a principal component analysis (PCA) (Fig. 3b). Two hundred and twenty-seven genes were differentially expressed between Ly5.1 and $\mathrm{Ltbr}^{-1-}$ LT/ST-HSCs 6 weeks post transplantation (Fig. 3c and Supplementary data 1). Gene set enrichment analysis (GSEA) revealed a downregulation of stemness- and apoptosis-related gene signatures, but an upregulation of stem cell proliferation, differentiation and cell cycling genes in $\mathrm{Ltbr}^{-/-}$vs. control LT/ST-HSCs (Fig. 3e). Gene expression analysis by quantitative reverse transcription PCR revealed an upregulation of genes involved in the cell cycle regulation and differentiation, such as $M y c, C c n d 1, C d k 4$, and Numb in the absence of LT $\beta R$ signaling. By contrast, stemness-related genes, such as those encoding for RNA-binding protein Musashi 2 (Msi2), Kit, and growth factor independent 1 transcriptional repressor (Gfil), were significantly downregulated (Supplementary Fig. 3a).

LIGHT-induced LT $\beta R$ signaling promotes apoptosis ${ }^{35}$. Similarly, $\mathrm{Ltbr}^{-1-}$ LT/ST-HSCs and LSKs expressed proapoptotic genes at lower levels and antiapoptotic genes at higher levels compared to Ly5.1 controls (Supplementary Fig. 3a).

In silico network and canonical pathway analysis from LSKs confirmed that LT $\beta R$ directly and indirectly regulates cell cycle, proliferation, apoptosis, and stemness-related pathways (e.g. Wnt, NF- $\kappa B$, regulators of HSC activity, and hematopoiesis) (Supplementary Fig. 3b). Interestingly, GSEA of $L t b r^{-1-}$ LT/ST-HSCs for signatures of both NF- $\mathrm{KB}$ pathways revealed significant alteration in the canonical NF- $\mathrm{kB}$ pathway but not in the signature of the noncanonical pathway (Fig. 3e). Correspondingly, Traf2 mRNA expression was lower in $\mathrm{Ltbr}^{-1-}$ vs. Ly5.1 LSKs (Supplementary Fig. $4 a, b)$.

Many of the TNFR ligands including LIGHT have been shown to provide reverse signaling ${ }^{36}$. LIGHT reverse signaling leads to the activation of MAPK/ERK and PI3K-AKT pathways ${ }^{37}$. GSEA revealed no differences in the signatures for MAPK, MAPK/ERK, or PI3K-AKT signaling (Supplementary Fig. 4c). Collectively, our data suggest that LT $\beta$ R regulates HSC stemness in stress-induced hematopoiesis through activation of the canonical NF- $\mathrm{kB}$ pathway.

LT $\beta$ R regulates HSC activity and promotes symmetric cell division. We next sought to functionally analyze the mechanisms by which LT $\beta$ R signaling regulates HSC function. To this end, we analyzed cell viability, cell cycle activity, and cell division of Ly5.1 and $L t b r^{-1-}$ HSCs in chimeric mice. Fewer $L t b r^{-1-}$ HSCs but not MPPs underwent apoptosis when compared to controls 6 weeks after transplantation (Fig. 4a). However, this difference disappeared 12 weeks after transplantation (Fig. $4 \mathrm{~b}$ ). 
a

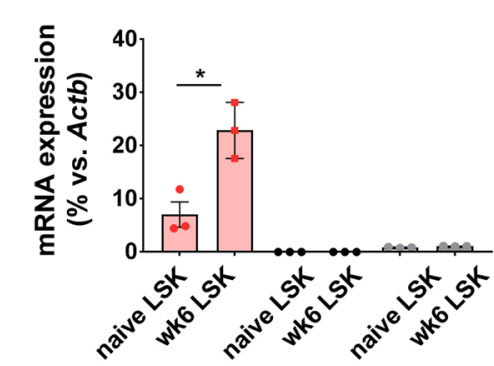

d

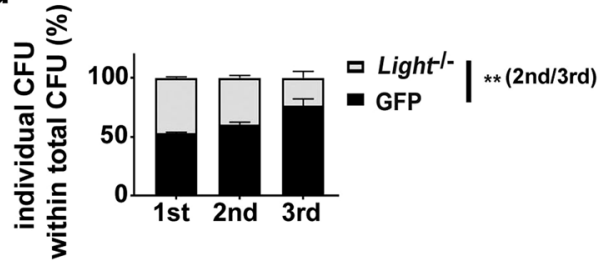

b

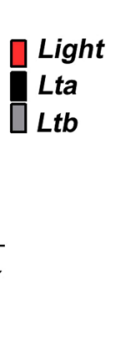

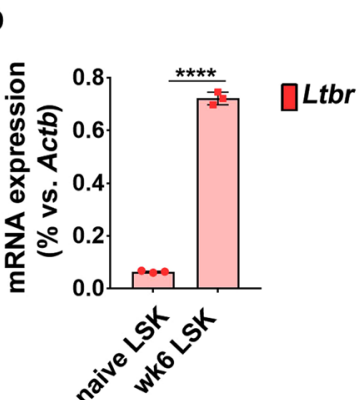

e
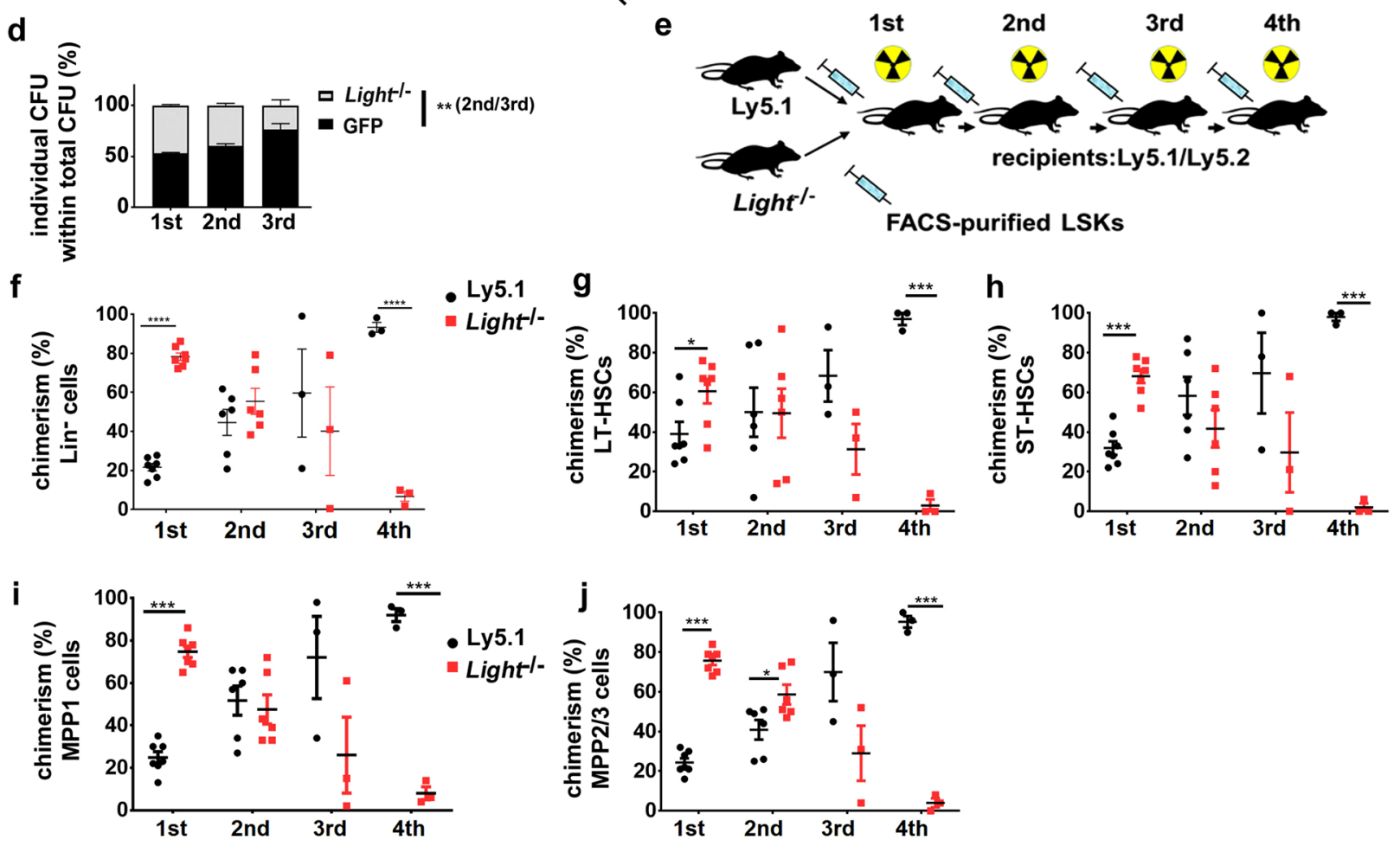

$\mathbf{k}$

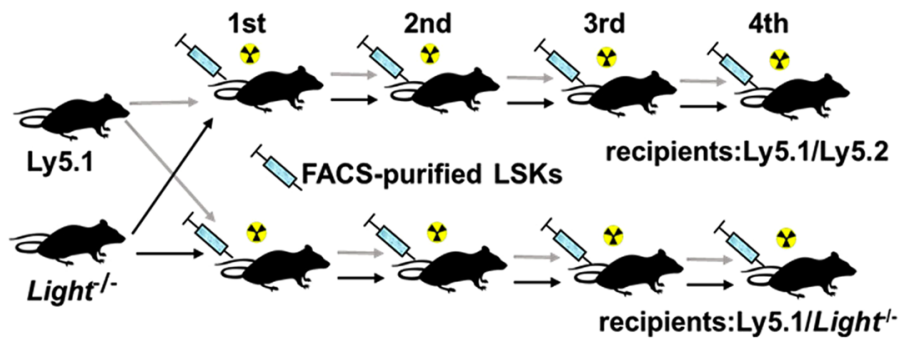

m

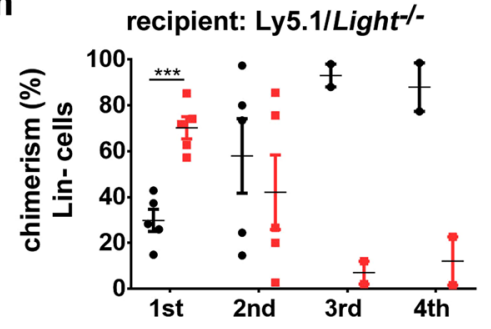

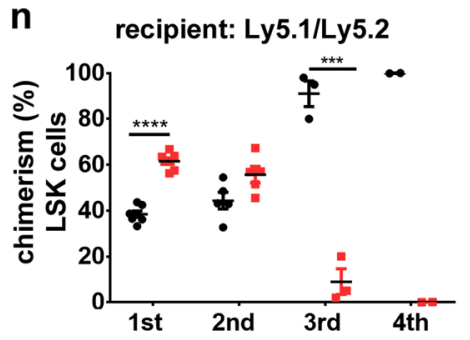

C

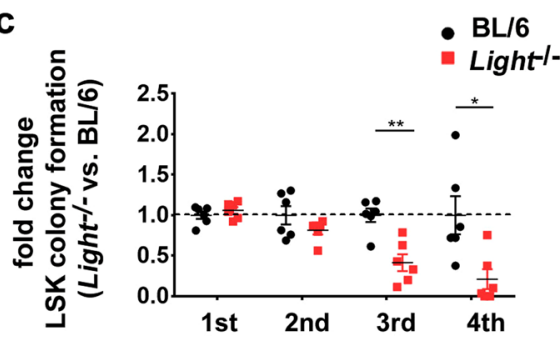

BL/6

Light-1- 
Fig. 2 LIGHT expressed by LSKs prevents exhaustion of HSCs. a Relative mRNA expression of Light (red), Lta (black), and Ltb (gray) by FACS-purified LSKs from naive mice $(n=3)$ and 1st chimeras 6 weeks post transplantation ( $n=4$ mice, two were pooled for the analysis). $\mathbf{b}$ Relative mRNA expression of Ltbr by FACS-purified LSKs from naive mice ( $n=3$ mice) and 1st chimeras 6 weeks after transplantation $(n=4$ mice, two were pooled for the analysis). c Fold change in colony formation of Light ${ }^{-/-}$BM LSKs (red) relative to BL/6 BM LSKs (black) in serial re-platings $(n=6)$. One out of two independent experiments is shown. d Percentage of CFU from Light ${ }^{-/-}$(gray) or BL/6- GFP LSKs (black) in mixed colony-forming assays. The frequency of GFP+

$(\mathrm{BL} / 6)$ vs. GFP- $\left(\right.$ Light $\left.^{-/-}\right)$colonies is shown. Data are pooled from two independent experiments and presented as mean \pm SEM. e Experimental scheme for serial transplantations of Light ${ }^{-/}-$Ly5.1 mixed BM chimeras. f-j Chimerism (red: Light ${ }^{-/}{ }^{-}$; black: Ly5.1) in percentages of donor Lin ${ }^{-}$cells (f), LT-HSCs $(\mathbf{g}), \mathrm{ST}-\mathrm{HSCs}(\mathbf{h}), \mathrm{MPP} 1(\mathbf{i}), \mathrm{MPP} 2 / 3\left(\mathrm{Lin}^{-} \mathrm{c}^{-} \mathrm{kit}^{+} \mathrm{sca}{ }^{+} \mathrm{CD} 150^{-} \mathrm{CD} 48^{+}\right)(\mathbf{j})$. Data are shown as mean $\pm \mathrm{SEM}$ of $n=7$ (1st), n=6 (2nd), n=3 (3rd, 4th) mice. $\mathbf{k}$ Experimental scheme of the serial transplantation of Ly5.1 and Light ${ }^{-/-}$LSKs (1:1) into lethally irradiated Ly5.1/Ly5.2 and congenic Ly5.1/Light ${ }^{-/-}$ recipients. I-o Percentages of donor Lin ${ }^{-}$cells in Ly5.1/Ly5.2 (I) and Ly5.1/Light-/- recipients (m) and LSKs in Ly5.1/Ly5.2 (n), and Ly5.1/Light ${ }^{-/-}$ recipients (o). Data are shown as mean \pm SEM, Ly5.1/Ly5.2 recipients: $n=7$ (1st), $n=5$ (2nd), $n=3$ (3rd); $n=3$ (4th) Ly5.1/Light ${ }^{-/-}$recipients: $n=5$ (1st), $n=5$ (2nd), $n=3$ (3rd), $n=3$ (4th). One out of two independent experiments is shown, Statistics: ${ }^{\star} p<0.05,{ }^{\star \star} p<0.01,{ }^{\star \star \star} p<0.001$, and ${ }^{\star \star \star \star} p<0.0001$ (two-tailed $t$ test), $\mathbf{a : ~} \mathrm{p}=0.005 ; \mathbf{b}: \mathrm{p}<0.0001 ; \mathbf{c}: \mathrm{p}=0.0014 ; \mathbf{d}: \mathrm{p}=0.014, \mathbf{f}: 1^{\text {st }} \mathrm{p}<0.0001,4$ th $p<0.0001 ; \mathbf{g}: 1$ st $p=0.0289$, 4th $p<0.0001$; h: 1st $p<0.0001$, 4th $p<0.0001$; i: 1st $p<0.0001$, 4th $p<0.0001 ; \mathbf{j}: p<0.0001,4$ th $p<0.0001 ;$ l: 1 st $p<0.0001$, 4th $p=0.043$; m: 1st $p=0.0004$, 4th $p<0.0001 ; \mathbf{n}: 1$ st $p<0.0001$, 3rd $p=0.0005 ; \mathbf{0}: p=0.0005$.

a

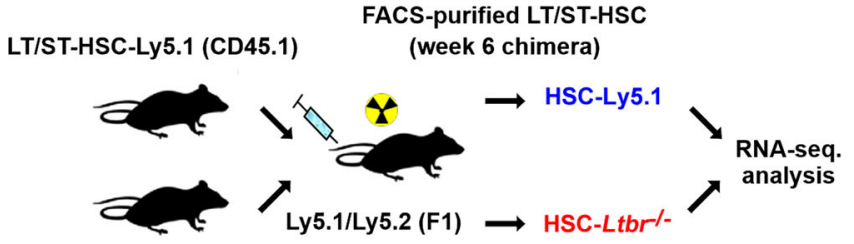

LT/ST-HSC-Ltbr/- (CD45.2)

C

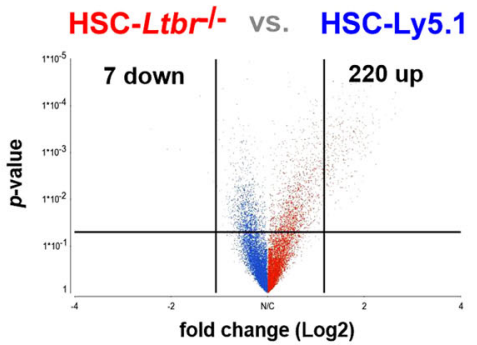

b

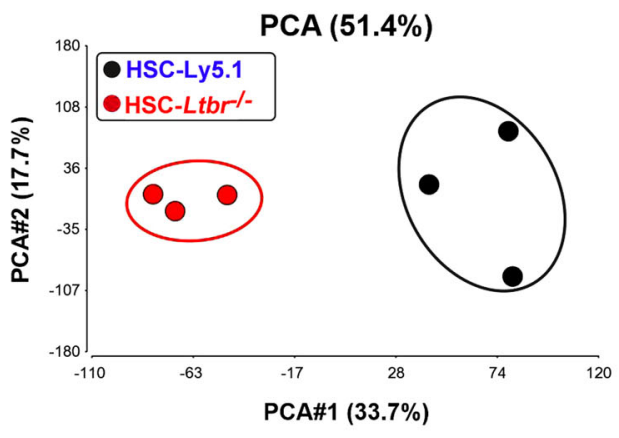

e

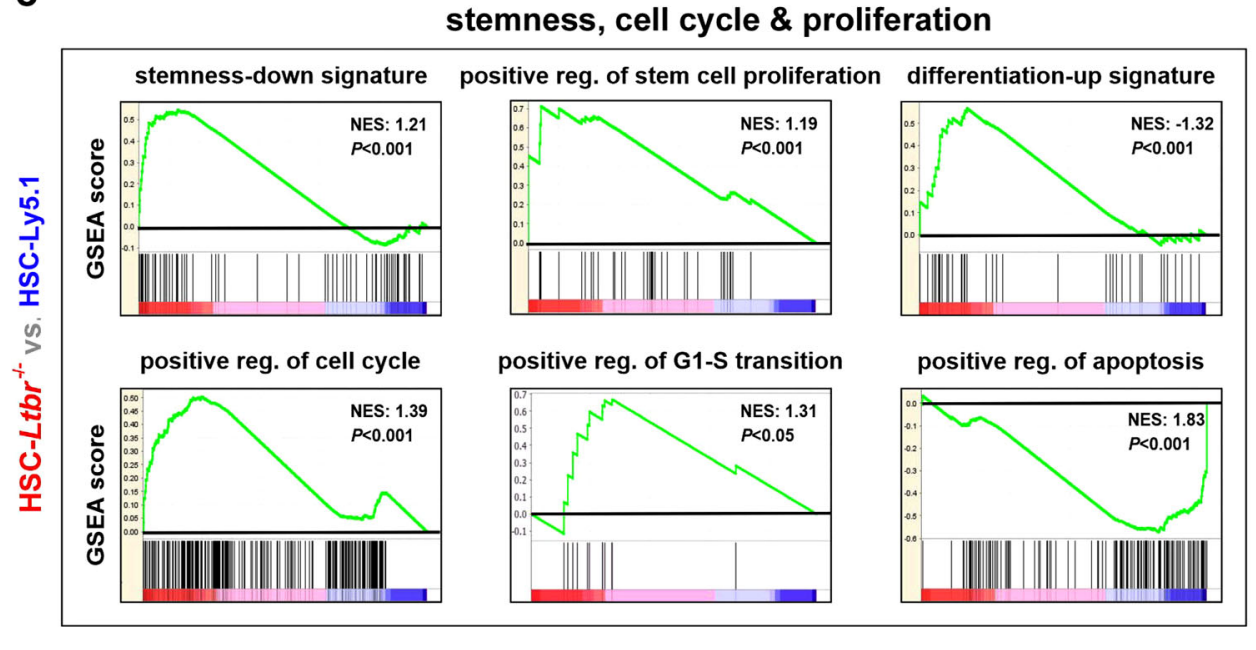

NFkB signaling

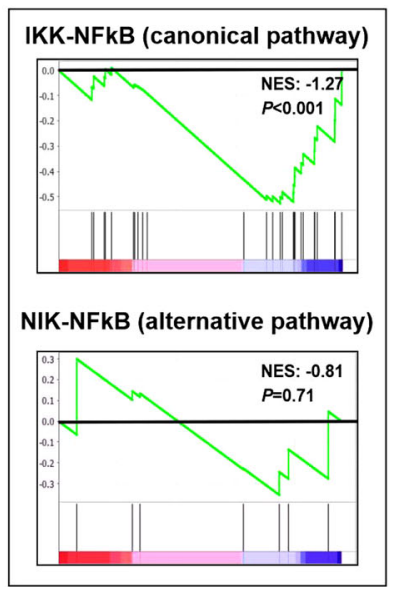

Fig. 3 Loss of LT $\beta R$ leads to a higher expression of genes related to cell cycling and survival while reducing the expression of genes defining stemness. a Experimental setting. FACS-purified $\mathrm{Ltbr}^{-/-}$and Ly5.1 LT/ST-HSCS from chimeras 6 weeks after primary transplantation ( $n=6$, pooled to three biological replicates) were used for RNA-seq analysis. b PCA with samples plotted using the 1st two principal components. $\mathbf{c}$ Volcano plot of differentially regulated genes. d Hierarchical clustering. e Gene set enrichment analysis (GSEA, pathcards.genecards.org). 
a

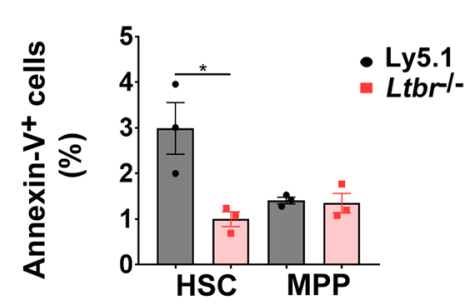

C

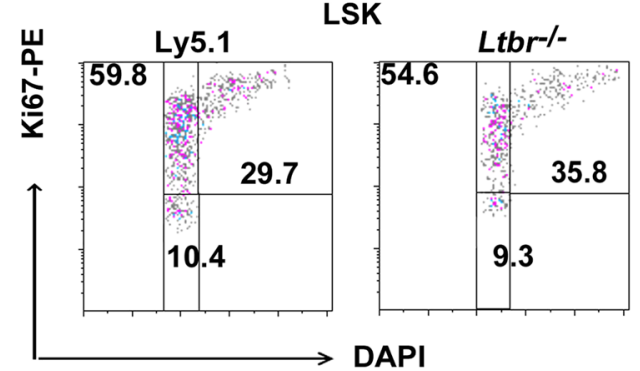

d $\quad\left[\begin{array}{l}\mathrm{G} 0 \\ \mathrm{G} 1 \\ \mathrm{G} 2-\mathrm{M}-\mathrm{S}\end{array}\right.$

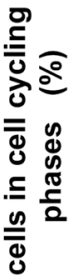

II

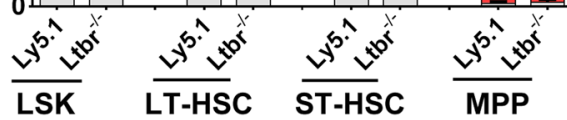

f

merge merge DAPI Tubulin (D/T) (T/N) Numb

merge bright
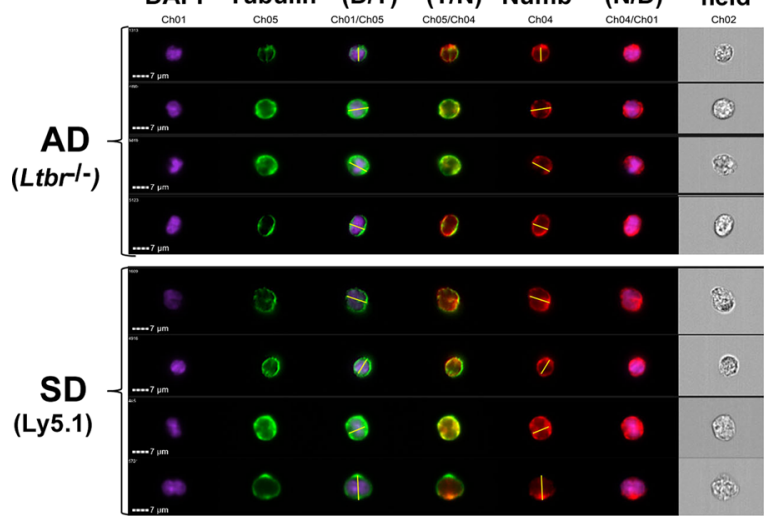

b
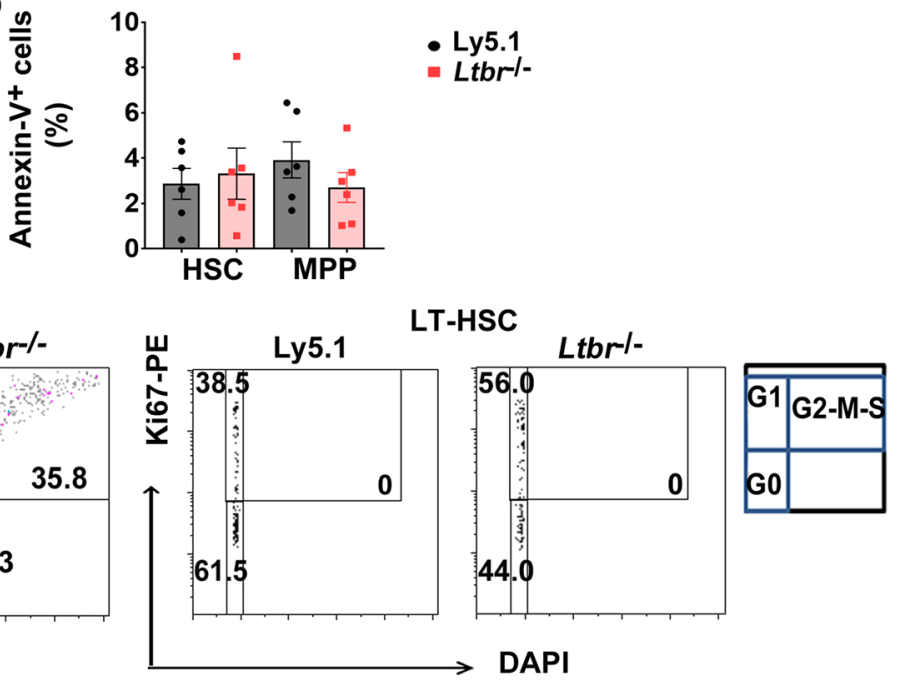

DAPI
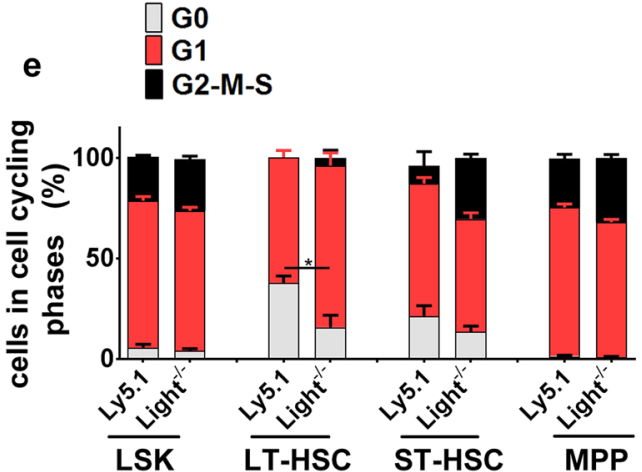

LSK

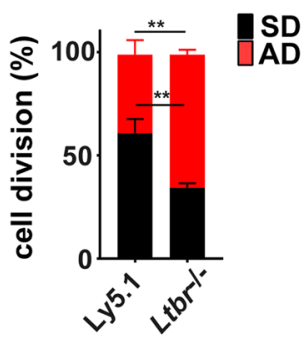

LT-IST-HSC

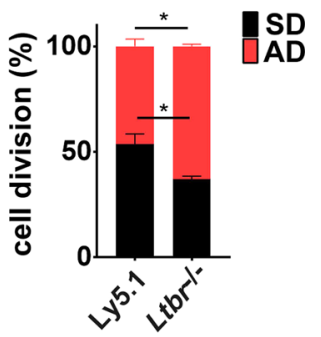

Fig. 4 Ltbr deficiency induces proliferation and differentiation of HSCs. a, b Percentages of Annexin-V+ HSCs and MPPs 6 (a) and 12 (b) weeks after $1 s t$ transplantation, black: Ly5.1, red: Ltbr ${ }^{-/-}$. One out of two independent experiments is shown, for $\mathbf{a}: n=3$, for $\mathbf{b}: n=6$ mice $\mathbf{c}$ Representative dot plot of the cell cycle analysis as determined by Ki67 and DAPI staining of Ly5.1 and Ltbr-/- BM LSKs and LT-HSCs 12 weeks after primary transplantation. Numbers in plots are shown as percentages of cells in each cell cycle phase, G0 in gray, G1 in red, and G2-M-S in black. d Frequency of Ly5.1 and Ltbr-/- LSKs, LTHSCs, ST-HSCs and total MPPs in different cell cycle phases 12 weeks post transplantation. Data are representative for two independent experiments, $n=$ 3. e Frequency of Ly5.1 and Light ${ }^{-/}-$LSKs, LT-HSCs, ST-HSCs, and total MPPs (CD48 $\left.{ }^{+}\right)$in different cell cycle status 12 weeks post transplantation. LSKs were isolated from two to three chimeric mice and pooled for the analysis. Pooled data from two independent experiments are shown. $\mathbf{f}$ Representative picture of Numb distribution in dividing Ly5.1 and Ltbr ${ }^{-/}$- FACS-purified LT/ST-HSCs 10-12 weeks after transplantation. DAPI in violet, $\alpha$-tubulin in green, and Numb in red. Plane cell division (yellow line) was assigned based on $\alpha$-tubulin and the cleavage furrow. Left: Quantification of Ly5.1 ( 92 cells from $n=$ 3 , pooled for the analysis) and Ltbr ${ }^{-/}-$LSKs ( 135 cells from $n=4$, pooled for the analysis) in SD (black) or AD (red). Right: Quantification of Ly5.1 (45 cells from $n=9$, pooled for analysis) and $L t b r^{-1}-L T / S T-H S C s$ (28 cells from $n=9$, pooled for analysis) in symmetric cell division (SD) or asymmetric cell division (AD). Cells were analyzed by ImageStreamX MkII. Nuclei were stained with DAPI. Data are shown as mean \pm SEM. Statistics: ${ }^{\star} P<0.05,{ }^{\star \star} P<0.01$ (two-tailed $t$ test), a: $p=0.028 ; \mathbf{f}(A D, S D$ right panel): $p=0.033$. 
setup (Fig. 4e). Taken together, these experiments indicate that LIGHT/LT $\beta$ R signaling maintains quiescence in primitive HSCs.

Re-entry of HSCs into cell cycle comprises the control between asymmetric and symmetric division that governs differentiation and maintenance of the stem cell pool ${ }^{6,12}$. To assess whether LT $\beta R$ signaling regulates the cell fate decision of HSPCs, we quantified asymmetric and symmetric cell divisions of Ly5.1 and $\mathrm{Ltbr}^{-1-}$ LSKs by analyzing the cell fate determinant Numb, a protein known to be distributed either symmetrically or asymmetrically in daughter cells during the division of stem/ progenitor cells. A higher Numb expression is associated with differentiation $2,40,41$. The expression of Numb showed a trend towards higher levels in $\mathrm{Ltbr}^{-1-}$ HSCs compared to Ly5.1 HSCs (Supplementary Fig. 5). The absence of LT $\beta$ R signaling in FACSpurified LSKs and LT/ST-HSCs promoted asymmetric over symmetric cell division (Fig. 4f). Symmetric cell division can theoretically lead to two stem cells (self-renewal) or to two differentiated daughter cells (commitment) ${ }^{28}$. Overall, this indicates that LIGHT/LT $\beta$ R signaling in primitive HSCs maintains self-renewal by regulating cell proliferation and asymmetric vs. symmetric cell division.

$\mathrm{LT} \beta \mathrm{R}$ regulates HSC function after genotoxic stress. Genotoxic drugs or irradiation lead to damage and eradication of hematopoietic cells and results in the activation of a demand-adapted hematopoiesis in order to replenish the hematopoietic system. To test hematopoietic reconstitution after genotoxic stress, we treated $\mathrm{BL} / 6$ and $\mathrm{Ltbr}^{-1-}$ mice with fluorouracil (5-FU) ${ }^{42}$. Since treatment with 5 -FU leads to downregulation of c-kit ${ }^{43}$, LSKs and all HSCs subsets were gated within the $\mathrm{Lin}^{-}$sca- $1^{+}$fraction (Supplementary Fig. 6a). 5-FU treatment resulted in increased $\mathrm{Ltbr}$ and Light mRNA and LTRR protein expression in LSKs (Fig. 5a-c). Interestingly, total BM cellularity was significantly higher in $\mathrm{Ltbr}^{-1-}$ compared to BL/6 mice 8 days after 5-FU treatment (Fig. 5d). In addition, the number of LT-HSCs was significantly increased in $\mathrm{Ltbr}^{-1-}$ compared to $\mathrm{BL} / 6$ mice, whereas the number of ST-HSCs and MPPs remained unchanged (Fig. 5e). In accordance with the reduced apoptosis rate of $\mathrm{Ltbr}^{-/}$HSCs in chimeric mice (Fig. 4a), significantly fewer $\mathrm{Ltbr}^{-/-}$HSCs and CD150+/CD48 ${ }^{+}$MPPs stained Annexin- ${ }^{+}$ compared to controls (Supplementary Fig. 6b). In addition, significantly fewer $\mathrm{Ltbr}^{-1-}$ LSKs were in the G0 phase of the cell cycle compared to BL/6 LSKs (Fig. 5f). Moreover, Ltbr deficiency resulted in an increase in asymmetric over symmetric cell division (Fig.5g). LSKs from $\mathrm{Ltbr}^{-1}-$ mice initially formed significantly more colonies 8 days after 5-FU treatment. Importantly, HSC and progenitors form colonies in the first plating. However, colony formation was consecutively lost in serial re-platings, suggesting a loss of HSCs with self-renewal capacity (Fig. 5h).

The phosphorylation and degradation of I $\kappa \mathrm{Ba}$ is a key process resulting in $\mathrm{NF}-\kappa \mathrm{B}$ activation ${ }^{44}$. I $\kappa \mathrm{B} \alpha$ was expressed at higher levels in naive and in 5-FU-treated $L_{b} r^{-/}$LT/ST-HSCs

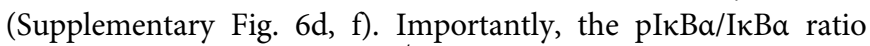
was similar in naive $L t b r^{-1-}$ LT/ST-HSCs but significantly reduced after 5-FU treatment when compared to control HSCs (Fig. 5i, j). This indicates that, in response to 5-FU treatment, LT $\beta \mathrm{R}$ signals via the canonical NF- $\kappa \mathrm{B}$ pathway.

Since LTa1 $\beta 2$ is an additional ligand for LT $\beta$ R and LIGHT also ligates herpesvirus entry mediator (HVEM), we analyzed a possible role of additional ligands/receptor in Light and $\mathrm{Ltbr}$ double knockout (KO) mice. Light ${ }^{-1-} / L_{t b r}-1-$ LSKs had a similar phenotype in colony formation compared to single $\mathrm{KO}$ LSKs, suggesting that LIGHT/LT $\beta$ R acts as a ligand/receptor pair and excluding a major role of other ligands/receptors (Supplementary Fig. 6g, h).
To study the number and function of HSCs after 5-FU treatment in vivo, we transplanted BM cells from 5-FU-treated Ly5.1 and $\mathrm{Ltbr}^{-1-}$ donors into lethally irradiated Ly5.1/Ly5.2 mice (Fig. 5k). $\mathrm{Ltbr}^{-1}-$ donor BM cells reconstituted recipient mice less efficiently than control BM cells, resulting in a significantly reduced frequency of $\mathrm{Lin}^{-}$cells and HSPCs (Fig. 5l). These findings suggest that the frequency of HSCs capable of reconstituting recipient mice is significantly lower in 5-FUtreated $\mathrm{Ltbr}^{-/}$mice than in controls. Together, these data indicate that LIGHT/LT $\beta$ R signaling reduces cell proliferation and asymmetric cell division and thereby maintains the pool of HSCs in response to genotoxic stress.

LIGHT/LT $\beta$ R signaling in LSCs promotes CML development. So far, our data indicated that LT $\beta$ R signaling regulates HSC cell cycling and self-renewal. Since self-renewal and regulation of cell fate in LSCs is crucial for the development of the disease, we next studied LT $\beta R$ signaling in a murine CML model ${ }^{45}$ CML-like disease was induced by injection of BCR-ABL1-GFP-transduced $\mathrm{BL} / 6$ or $\mathrm{Ltbr}^{-1-}$ LSKs into nonirradiated BL/6 recipients (Fig. 6a). In this model, LSCs are characterized as $\mathrm{GFP}^{+}$LSKs and LSC subsets are defined in analogy to normal HSC subpopulations ${ }^{18}$ (Supplementary Fig. 7a).

LSCs expressed Ltbr mRNA at significantly higher levels than normal LSKs (Fig. 6b). FACS analysis revealed that LT $\beta$ R was expressed on LSCs and leukemia progenitors with the highest expression on LT-LSCs (Fig. $6 \mathrm{c}$ and Supplementary Fig. 7b-d). In addition, LSCs expressed Light at higher levels than normal LSKs (Fig. 6d). tbr $^{-1-}$ CML progressed significantly slower than BL/6 $\mathrm{CML}$ with reduced leukemia granulocytes in $\mathrm{PB}$ leading to a prolonged survival (Fig. 6e, f). In addition, spleen weights of $\mathrm{Ltbr}^{-1}$ - CML mice were significantly reduced compared to BL/6 CML mice 19 days post transplantation, indicating a lower leukemia burden (Fig. 6g). Importantly, significantly fewer LSCs (LT- and ST-LSCs) and MPPs were found in the BM of $\mathrm{Ltbr}^{-1-}$ compared to BL/6 CML mice 19 days after transplantation (Fig. 6h-j).

In order to functionally analyze the role of LT $\beta R$ signaling in LSCs, we transplanted either whole BM or FACS-purified LSCs from primary $\mathrm{BL} / 6$ and $L t b r^{-1}-\mathrm{CML}$ mice into nonirradiated BL/6 recipients (Fig. 6k). Recipients of $L t b r^{-1-}$ CML BM (Fig. 6l) or LSCs (Fig. $6 \mathrm{~m}$ ) survived significantly longer than recipients of $\mathrm{BL} / 6 \mathrm{BM}$ or LSCs, respectively. This indicates that $\mathrm{Ltbr}^{-1-} \mathrm{CML}$ harbors fewer LSCs and that the phenotypically characterized $\mathrm{Ltbr}^{-1-}$ LSCs are functionally impaired.

Lack of LT $\beta$ R on LSCs increased cell cycle activity with an $\sim 2$ fold decrease of LT-LSCs in G0 phase compared to BL/6 LT-LSCs $(14.7 \pm 10.2$ vs. $33.4 \pm 11.6)$. By contrast, cell cycle activity of ST-LSCs or MPPs did not depend on LT $\beta$ R signaling (Fig. 6n). Moreover, the analysis of the distribution of Numb in dividing LSCs in telophase revealed an increased number of $L t b r^{-/-}$LSCs in asymmetric over symmetric cell division (Fig. 6o). Moreover, Numb expression is higher in $L t b r^{-1-}$ LSCs compared to BL6 LSCs (Supplementary Fig. 7e).

Similarly, CML induced by Light $^{-1-}$ BCR-ABL1-GFP LSKs (Light ${ }^{-1-}$ CML) progressed significantly slower than BL/6 CML with reduced numbers of leukemia granulocytes in $\mathrm{PB}$ and significantly reduced spleen weights 18 days post transplantation (Fig. 6p, q). In addition, significantly fewer LSCs (LT- and STLSCs) and MPPs were found in the BM of Light $^{-1-}$ compared to $\mathrm{BL} / 6 \mathrm{CML}$ mice 18 days post transplantation (Fig. 6r-t). Supplementation of recombinant LIGHT in the colony-forming assay did not rescue the KO phenotype (Supplementary Fig. 7f). This finding is in agreement with the documented role of cellautonomous LIGHT/LT $\beta$ R (cis) signaling. These data indicate 

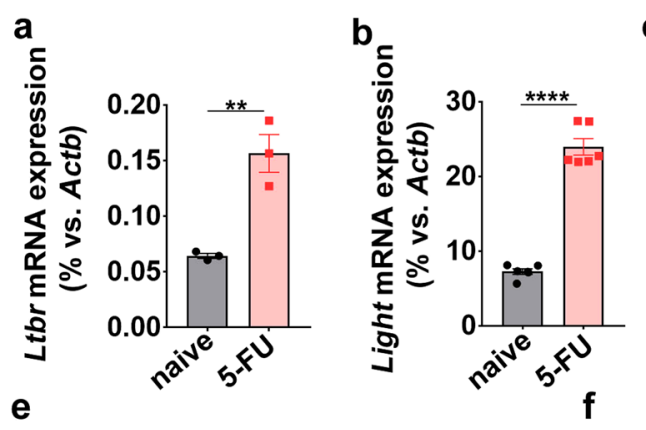

C

d
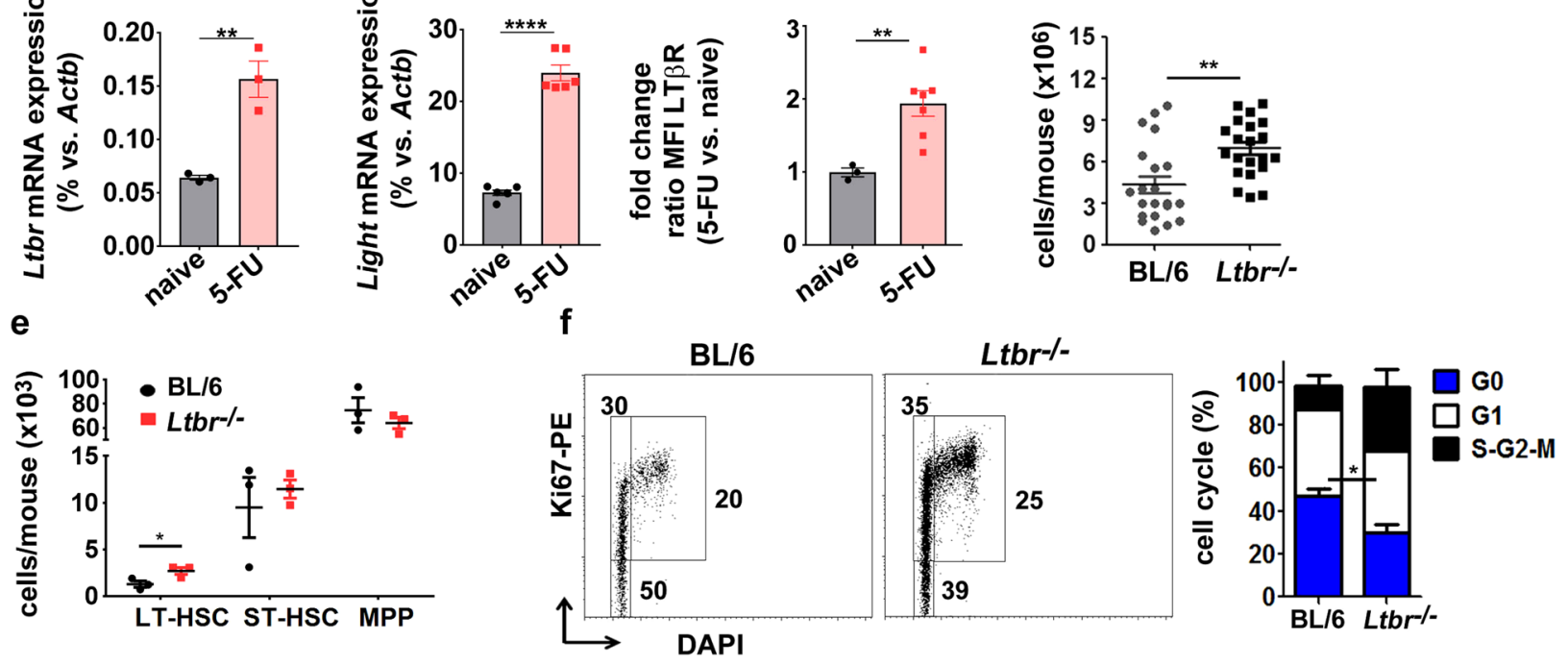

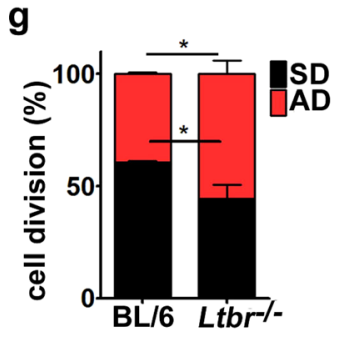

$\mathbf{k}$

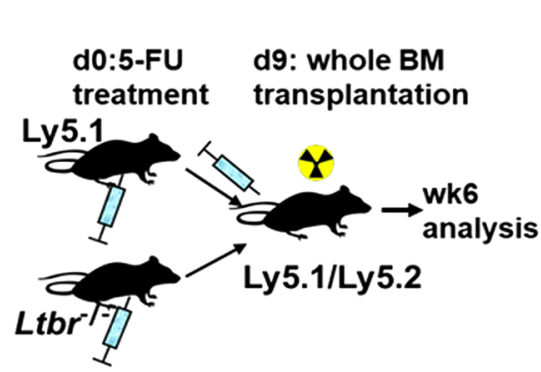

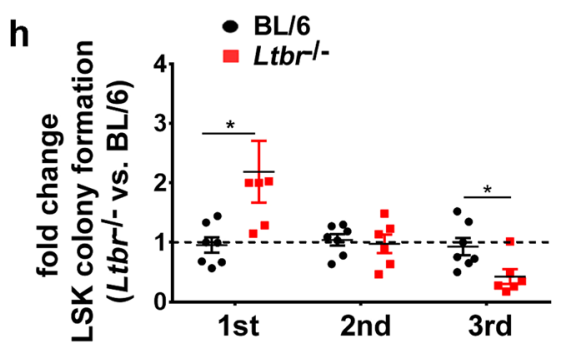

I

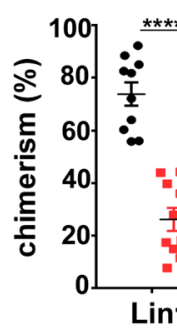

i

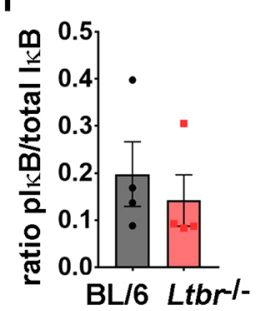

j

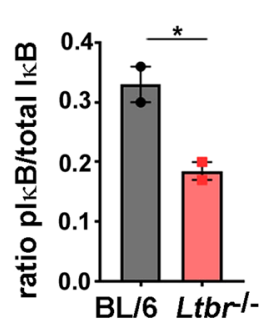

- Ly5.1

- Ltbr-1-

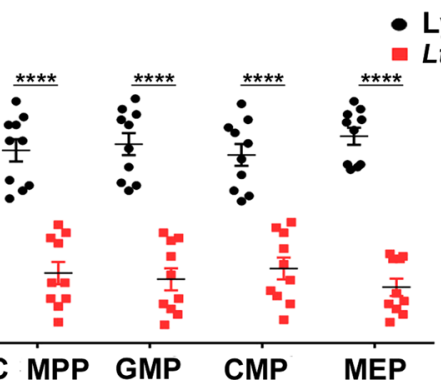

Fig. 5 Treatment of $\mathbf{L t b r}^{-\boldsymbol{I}}$ - mice with 5-FU leads to increased HSC cell cycle activity and reduced long-term repopulation. a, b Quantitative RT-PCR analysis of Ltbr (a) and Light (b) in BM LSKs from naive (black) and 5-FU-treated (red) BL/6 mice 8 days after treatment (for Ltbr: $n=3$, for Light: $n=6$ ). c Fold change of LT $\beta R$ MFI on HSCs from 5 -FU-treated BL/6 (red, $n=7$ ) vs. naive BL/6 mice (black, $n=3$ ). d Total BM cell numbers of BL/6 (gray) and $\mathrm{Ltbr}^{-/-}$mice (black) 8 days post 5 -FU injection $\left(\mathrm{BL} / 6 n=20, \mathrm{Ltbr}^{-/-} n=21\right)$. e Total numbers of BM LSK subpopulations in 5-FU-treated BL/6 (black) and $L t b r^{-/}$mice (red), $n=3$. $\mathbf{f}$ Left: representative FACS profiles of cell cycle analysis of BM LSKs. Right: Percentage of BL/ 6 and $L t b r-/-L S K s$ in each cell cycle phase, blue: G0, white: G1, black: S-G2-M. Data represent one out of two independent experiments; LSKs were pooled for analysis from BL/6 $n=3$ and $\mathrm{Ltbr}^{-/-} n=5$ mice. $\mathbf{g}$ Percentages of FACS-purified LSKs from 5-FU-treated BL/6 and $\mathrm{Ltbr}^{-/}$- mice in symmetric (SD, black) or asymmetric division $\left(A D\right.$, red). Data represent one out of two independent experiments, LSK numbers $n=73(\mathrm{BL} / 6)$ and $n=85\left(\mathrm{Ltbr}^{-/-}\right)$. $\mathbf{h}$ Fold change of CFU capacity of FACS-purified BL/6 (black) and $\mathrm{Ltbr}^{-/}$- (red) LSKs in serial re-plating experiments, pooled data from two independent experiments are shown (BL/6 $n=$ 5 , $L t b r^{-/-} n=7$ ). i Ratio of $1 \kappa B \alpha$ protein expression and its phosphorylation level of BM LT/ST-HSCs from naive BL/6 (black) and Ltbr ${ }^{-/-}$mice (red). Data were pooled from two independent experiments (BL/6 $n=5$ mice, $L t b r^{-/}-n=4$ mice). $\mathbf{j}$ Ratio of $\mid \kappa B \alpha$ protein expression and its phosphorylation level of

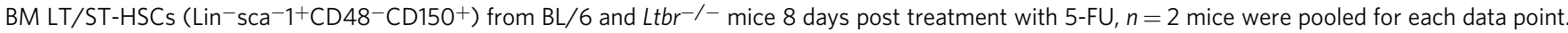
One out of two independent experiments is shown. $\mathbf{k}$ Experimental setup of BM reconstitution after 5-FU treatment. I Percentages of Ly5.1 (black) and $\mathrm{Ltbr}^{-/}$- BM donor cells (red) of recipients 6 weeks post transplantation, one out of two independent experiments $(n=10)$. Unless otherwise stated, data are presented as mean $\pm \mathrm{SEM},{ }^{\star} P<0.05,{ }^{\star \star} p<0.01,{ }^{\star \star \star \star} P<0.0001$ (two-tailed $t$ test). a: $p=0.0058 ; \mathbf{b}: p<0.0001 ; \mathbf{c}: p=0.0092 ; \mathbf{d}: 0.0039 ; \mathbf{e}: p=0.030$; f: $0.025 ; \mathbf{g}: p=0.042 ; \mathbf{h}: 1$ st $p=0.031$, 3rd $p=0.025 ; \mathbf{i}: p=0.55 ; \mathbf{j}: p=0.047 ; \mathbf{l}: p<0.0001$.

that LIGHT/LT $\beta$ R signaling regulates cell division and cell fate in LSCs and promotes disease progression.

LT $\beta R$ signaling regulates stemness in human $\mathrm{CD}^{+} 4^{+}$HSPCs. LTBR and LIGHT are expressed by human CD34 ${ }^{+} \mathrm{BM}$ cells on the mRNA level (GEO: GSE32719) ${ }^{46}$ (Fig. 7a). Next, we silenced LT $\beta R$ expression in FACS-purified BM CD34 ${ }^{+}$HSPCs from untreated staging negative lymphoma patients (control samples) using small interfering RNA (siRNA) (Supplementary Fig. 8a). $L T B R$ knockdown significantly increased the expression of genes related to proliferation, such as CCND1, but reduced 
a

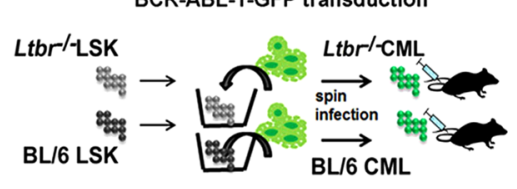

b

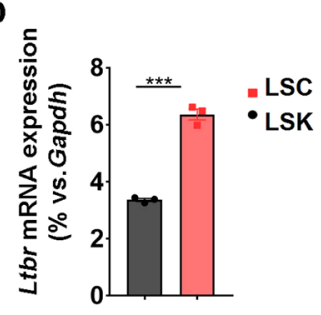

C

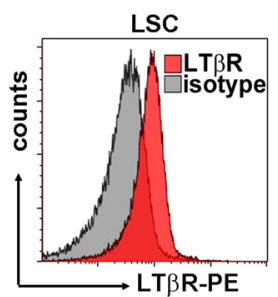

g

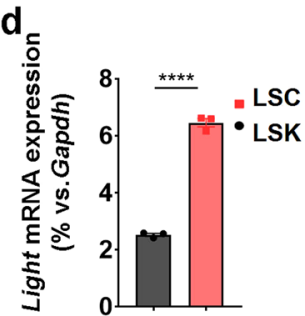

h

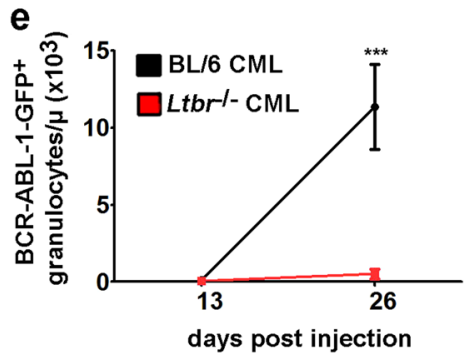

f

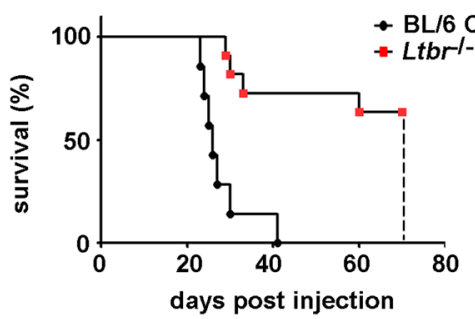

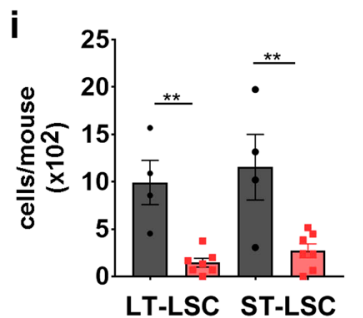

m

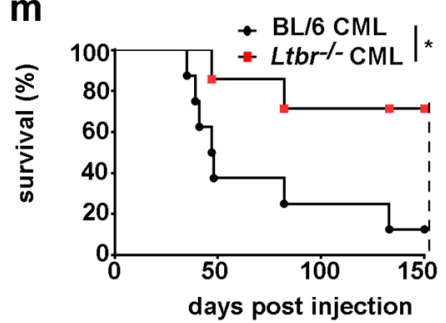

k

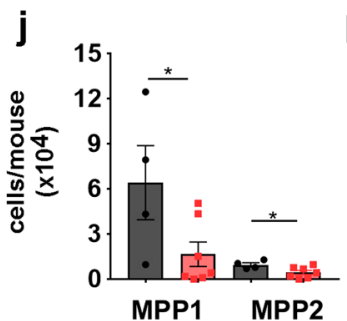

n

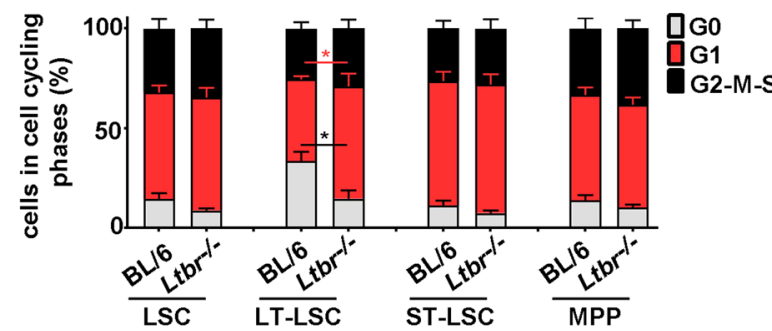

k
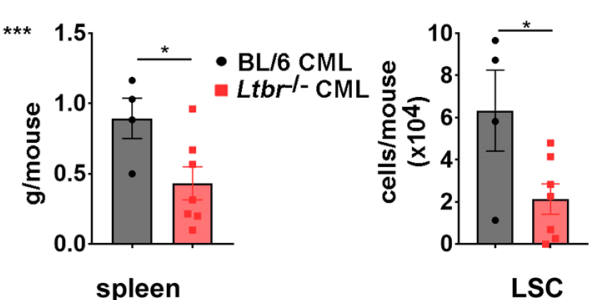

1st Ltbr' CML
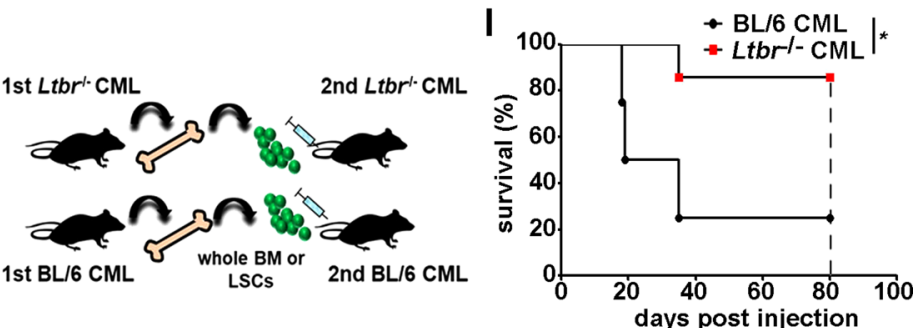

。

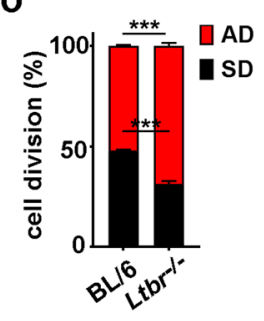

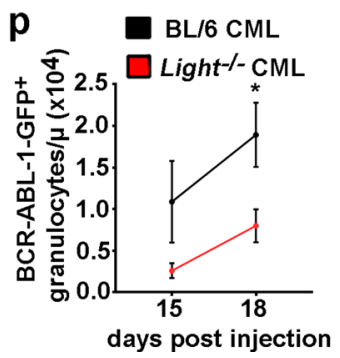

days post injection q $\bullet B L / 6 \mathrm{CML} \quad \mathbf{r}$

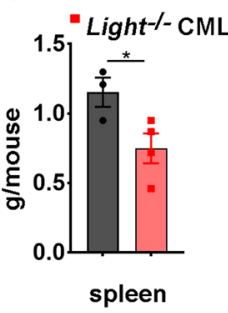

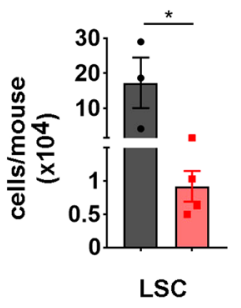

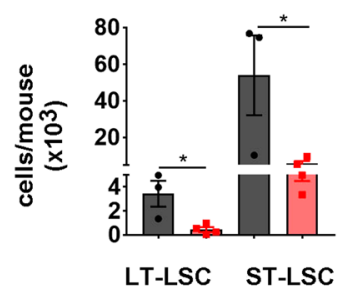

$\mathbf{t}$

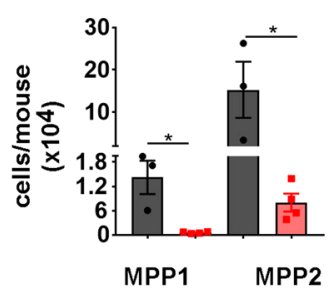

stemness-related gene such as MSI2 (Fig. 7b). In addition, CD34+ $\mathrm{BM}$ cells treated with siRNA for $24 \mathrm{~h}$ formed significantly fewer colonies in methylcellulose and lost the capacity to form colonies in serial re-platings, indicating that the knockdown of $L T B R$ reduced the number of functional HSCs (Fig. 7c).

To analyze the role of LIGHT/LT $\beta$ R signaling in activated hematopoiesis, we isolated CD $34^{+}$HSPCs from PB of patients that have been treated with cyclophosphamide mobilization chemotherapy and granulocyte colony-stimulating factor (G-CSF). Interestingly, LT $\beta$ R and LIGHT expression were increased in GCSF-mobilized CD34 ${ }^{+}$HSPCs compared to control BM CD34 ${ }^{+}$ HSPCs (Fig. 7d). LTBR knockdown in G-CSF-mobilized CD34 ${ }^{+}$ HSPCs reduced colony-forming unit (CFU) capacity in the first, second, and third plating in methylcellulose. By contrast, LIGHT
siRNA treatment resulted in similar numbers of colonies in the first plating, but significantly fewer colonies after re-plating (Fig. 7e). As expected, siRNA treatment did only transiently downregulate LT $\beta$ R and LIGHT with reconstituted expression after the first plating (Supplementary Fig. 8d). This indicates that transient silencing of LT $\beta$ R or LIGHT leads to differentiation in the first plating with fewer HSCs capable of self-renewing in the second and third re-plating. $L T B R$ and LIGHT knockdown significantly reduced the expression of the stemness-related gene MSI2 in GCSF-mobilized HSPCs (Fig. 7f). Importantly, the expression of MSI2 positively correlated with the expression of LTBR and LIGHT after knockdown (Supplementary Fig. 8e, f). To analyze the expression of LTBR, LTA, LTB, and LIGHT in CD34+ CML stem/ progenitor cells, we took advantage of a public available microarray 
Fig. 6 LT $\beta R$ expression of LSCs promotes CML progression. a Schematic for CML induction. $\mathbf{b}$ Ltbr ${ }^{-/-}$mRNA expression in naive BL/6 LSKs (black) and $\mathrm{LSCs}$ (red, $\mathrm{Lin}^{-} \mathrm{GFP}^{+}{ }^{\mathrm{c}-\mathrm{kit}}{ }^{+} \mathrm{sca} 1^{+}, n=3$ ). $\mathbf{c ~ L T} \beta R$ expression on BL/ $6 \mathrm{LSCs}$. $\mathbf{d}$ Light mRNA expression of naive BL/ $6 \mathrm{LSKs}$ (black) and BL/6 LSCs (red, $n=3$ ). e BCR/ABL1-GFP+ granulocytes/ $\mu$ in blood from BL/6 (black, $n=12$ ) and $L t b r^{-/}-C M L$ mice $(r e d, n=13$ ). One out of two independent experiments is shown. f Kaplan-Meier survival curves of BL/6 $(n=7)$ and $L t b r^{-1}-\mathrm{CML}$ mice $(n=11$, dashed line: predetermined endpoint of the experiment). $\mathbf{g}$ Spleen weight of BL/6 (black, $n=4$ ) and Ltbr ${ }^{-/}-\mathrm{CML}$ mice (red, $\left.n=7\right)$. $\mathbf{h}-\mathbf{j}$ LSC (h), LT-LSC, ST-LSC (i), and leukemic MPPs (j) cell numbers in BM of BL/6 and $\mathrm{Ltbr}^{-/-}$mice. Data are shown as one out of three independent experiments. $\mathbf{k}$ Schematic of secondary CML transplantation with BM cells or LSCs.

I, $\mathbf{m}$ Kaplan-Meier survival curves for whole CML BM cells (I, BL/6 $\left.n=4, \mathrm{Ltbr}^{-/-} n=7\right)$ and LSCs ( $\left.\mathbf{m}, \mathrm{BL} / 6 n=8, \mathrm{Ltbr}-/-n=7\right)$. $\mathbf{n}$ Percentage of LT-HSC, ST-HSC, and MPPs from BL/6 and $\mathrm{Ltbr}^{-/-}$mice in GO (gray), G1 (red), and G2-M-S (black). Data are pooled from two independent experiments ( $n=6$ ). - Percentages of $\mathrm{BL} / 6$ and $\mathrm{Ltbr}^{-/}$- LSCs in symmetric (SD, black) or asymmetric division (AD, red), $n=140$ cells (BL/6) and $n=230$ cells ( $\left(\mathrm{Ltbr} r^{-/-}\right.$) were examined in two independent experiments. Data are shown as mean \pm SEM. $\mathbf{p}$ BCR/ABL1-GFP + granulocytes $/ \mu$ in blood from BL/6 (black, $n=6)$ and Light $^{-/}-\mathrm{CML}$ mice (red, $n=8$ ). One out of two independent experiments is shown. q Spleen weight of BL/6 (black, $n=3$ ) and Light ${ }^{-/-} \mathrm{CML}$ mice (red, $n=4)$. $\mathbf{r}-\mathbf{t}$ LSC $(\mathbf{r})$, LT-LSC, ST-LSC $(\mathbf{s})$, and leukemic MPPs $(\mathbf{t})$ cell numbers in BM of BL/6 $(n=3)$ and Light ${ }^{-/-}$CML mice $(n=4)$. Data are shown as mean \pm SEM. Statistics: ${ }^{\star} P<0.05,{ }^{\star \star} P<0.01,{ }^{\star \star \star} P<0.001$, and ${ }^{\star \star \star \star} P<0.0001$ (two-tailed $t$ test: $\mathbf{b}, \mathbf{d}, \mathbf{e}, \mathbf{g}, \mathbf{h}-\mathbf{j}, \mathbf{n}-\mathbf{p}, \mathbf{q}-\mathbf{t}$ ), two-tailed log-rank test (f, $\mathbf{l}, \mathbf{m}$ ), b: $p=0.0001 ; \mathbf{d}: p<0.0001 ; \mathbf{e}: p=0.0001 ; \mathbf{f}: p=0.0002 ; \mathbf{g}: p=0.038 ; \mathbf{h}: p=0.036 ; \mathbf{i}:$ LT-LSC $p=0.0011 ;$ ST-LSC $p=0.0095 ; \mathbf{j}$ MPP1 $p=0.049$, MPP2 $p=0.04 ; \mathbf{I}: p=0.028 ; \mathbf{m}: p=0.0196 ; \mathbf{n}: \mathrm{GO} p=0.014, \mathrm{G} 1 p=0.044 ; \mathbf{o}: \mathrm{SD} / \mathrm{AD} p=0.0003 ; \mathbf{p}: p=0.021 ; \mathbf{q}: p=0.048 ; \mathbf{r}: p=0.043 ; \mathbf{s}: \mathrm{LT}-\mathrm{LSC} p=0.024$ ST-LSC $p=0.048 ; \mathbf{t}:$ MPP1 $p=0.012$, MPP2 $p=0.048$.
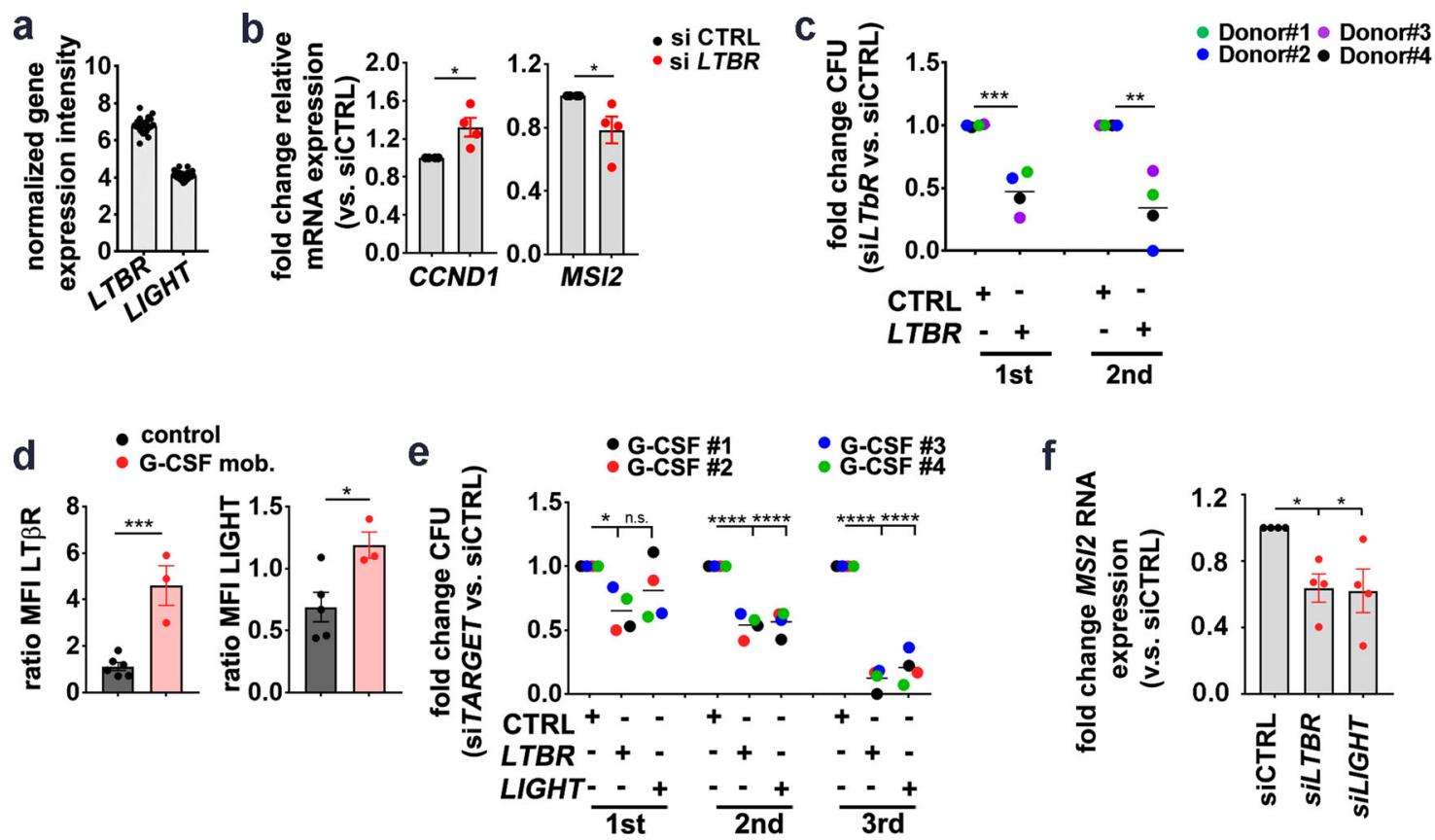

Fig. 7 LT $\beta$ R signaling in human HSPCs. a mRNA expression intensity of $L T B R$ and $L I G H T$ in CD34+ cells from 27 healthy donors, analyzed in a publicly available microarray dataset (GSE32719). b Fold change of relative mRNA expression of indicated genes in HSPCS (CD45 int Lin ${ }^{-}$CD34 ${ }^{+}$) from control samples, transfected with siLTBR (red) relative to HSPCs treated with siCTRL (black), $n=4$, pooled for analysis from three independent experiments. c Fold-change CFU and re-plating of siLTBR- or siCTRL-transfected BM HSPCs ( $n=4$, pooled for analysis from two independent experiments, shown as grand mean). d MFI (stain/isotype) of LT $\beta R$ and LIGHT in BM HSPCs from control samples (black, $n=6$ ) and G-CSF-mobilized PB HSPCs (red, $n=3$ ). Data from five independent experiments were pooled for analysis. e Fold-change CFU and re-plating of PB HSPCs from G-CSF-treated patients, transfected with indicated siRNA $(n=4)$. Data are pooled for analysis from two independent experiments, shown as grand mean. $\mathbf{f}$ Fold change of MSI2 mRNA in HSPCs from G-CSF patients, transfected with siLTBR (red) or siLIGHT (red) relative to HSPCs treated with siCTRL (black) ( $n=4$, pooled data for analysis from two independent experiments). Data are presented as mean \pm SEM. Statistics: ${ }^{\star} P<0.05,{ }^{\star \star} P<0.01,{ }^{\star \star \star} p>0.001$ (two-tailed $t$ test: $\mathbf{b}$-d, and one-way ANOVA: e, f). b: CCND1 $p=0.018$, MSI2 $p=0.043$; c: 1st $p=0.0007$, 2nd $p=0.0028$; d: LT $\beta R p=0.0007$, LIGHT $p=0.030$; e: 1st $p=0.04,2$ nd $p<0.0001,3 r d p<0.0001 ;$ f: CTRL vs. siLTBR $p=0.035$, CTRL vs. siLIGHT $p=0.029$. n.s. Not significant.

dataset (GEO: GSE11675) ${ }^{47}$. While $L T B$ was similarly expressed in normal and in CML CD34 ${ }^{+}$cells, LIGHT and LTBR expression was clearly increased in CML samples (Fig. 8a). In accordance, FACS analysis of CD34 ${ }^{+}$HSPCs from CML patients revealed a significantly higher LIGHT expression and a trend to a higher expression of LT $\beta \mathrm{R}$ than in control CD34 ${ }^{+}$HSPCs (Fig. 8b). LTBR knockdown in CD34+ ${ }^{+} \mathrm{CML}$ cells resulted in more colonies in the first plating in methylcellulose but fewer colonies in the second replating (Fig. 8c and Supplementary Fig. 8g). Importantly, knockdown of LTBR reduced the expression of MSI2, CTNNB1, and TNIK, indicating reduced stemness (Fig. 8d).
LIGHT knockdown in CML CD34 ${ }^{+}$HSPCs resulted in a reduced CFU capacity after re-plating (Fig. 8e and Supplementary Fig. 8h). Collectively, these data indicate that LIGHT/LT $\beta$ R signaling contributes to the maintenance and self-renewal of human hematopoietic and CML stem/progenitor cells.

\section{Discussion}

In response to an increased demand of blood cells, HSPCs are activated to enter cell cycling, proliferation, differentiation, and migration ${ }^{48}$. This activation is regulated by paracrine signals from 
a

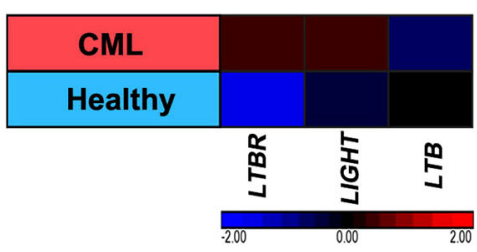

b

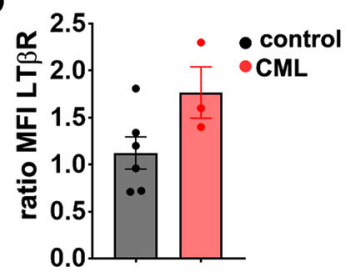

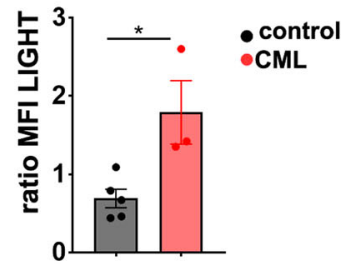

$\mathbf{e}$

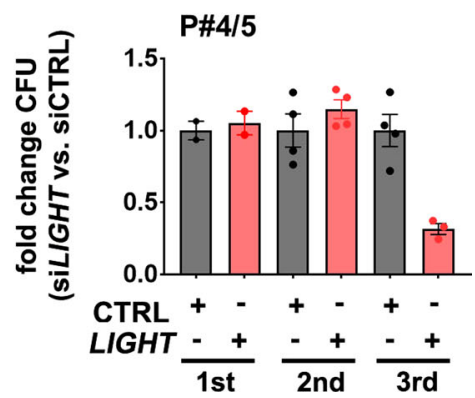

Fig. 8 LT $\beta$ R signaling in CD34 ${ }^{+}$HSPCs from CML patients. a Heatmap analysis for LTBR, LIGHT, and LTB gene expression (log 2 fold differences) in PB CD34+ Lin $^{-}$cells from CML patients (red) and healthy donors (blue) of a publicly available dataset (GEO: GSE11675). $\mathbf{b}$ MFI of LT $\beta R$ and LIGHT in BM HSPCs (black, $n=6$ ) and BM HSPCs from CML patients (red, $n=3$ ). Data are pooled for analysis from five independent experiments. c Fold change CFU and re-plating of CML HSPCs, transfected with siLTBR vs. siCTRL $(n=4$, one sample (Pat 4/5) was used as pooled cells from two CML patients. Data are pooled for analysis from two independent experiments and shown as grand mean. d Fold change of mRNA expression of indicated genes in siLTBR (red)transfected CML HSPCs, relative to siCTRL (black)-treated CML HSPCs ( $n=4$, data are pooled for analysis from two independent experiments. e Foldchange CFU and re-platings of siLIGHT (red)-transfected CML HSPCs, relative to siCTRL (black). Cells were pooled from $n=2 \mathrm{CML}$ patients. Data are presented as mean \pm SEM. Statistics: ${ }^{\star} P<0.05$ (two-tailed $t$ test: $\mathbf{b}, \mathbf{c}$ ). $\mathbf{b}$ : LT $\beta R, p=0.07$, LIGHT, $p=0.030 ; \mathbf{c}: 1$ st, $p=0.018,2$ nd, $p=0.029$.

the HSC niche cells including ECs and BM MSCs and from immune cells of the BM microenvironment ${ }^{6,48}$. For example, type I and type II IFNs, G-CSF, and LPS are important activators of the hematopoiesis in response to increased demand ${ }^{10}$. In addition, HSCs sense pathogen-associated molecular patterns during infection mainly via TLRs and respond with proliferation and myeloid differentiation ${ }^{49}$. Danger signals such as TLR signaling have been shown to induce the autocrine production of hematopoietic cytokines in HSPC ${ }^{50}$. However, primitive HSCs cannot be activated to proliferate indefinitely since this will lead to exhaustion of HSCs and loss of LT hematopoiesis ${ }^{51}$. Therefore, signaling pathways that regulate HSCs quiescence and cell fate are crucial for the maintenance of the HSC pool. More than 200 genes have been identified to regulate HSC function ${ }^{52}$. They are mainly involved in the regulation of cell cycling, Pten/Akt, and Wnt pathway ${ }^{53-55}$. In addition, external cues maintain HSC quiescence and self-renewal.

Different TNFRs have been implicated in the regulation of hematopoiesis. TNF- $\alpha$ is a major regulator of demand-adapted hematopoiesis via signaling through the p55 TNFR $1 a^{56}$. CD 40L stimulates human cord blood HSPC proliferation and myeloid differentiation ${ }^{57}$. In addition, CD70-expressing immune cells regulate HSPC function and differentiation during infection via CD27 signaling ${ }^{16,17}$. CD27, CD40, and LT $\beta$ R signal via TRAFs 2 and 5 , which activate multiple signaling pathways, including the $N F-\kappa B$ pathway ${ }^{58-60}$. Our GSEA and a conformational analysis of the phosphorylation status of I $\mathrm{KB} \alpha$ revealed that LIGHT/LT $\beta R$ preferentially signals via the canonical NF- $\kappa B$ pathway. Importantly, the NF- $\mathrm{KB}$ pathway has been documented before as a central regulator of HSC maintenance and homeostasis ${ }^{61}$.

In addition, CD27 signaling contributes to the maintenance and expansion of LSCs via TRAF2/TNIK signaling and Wnt pathway activation ${ }^{18}$. CD27 and LT $\beta R$ signaling is mainly regulated by the expression of its respective ligands. The CD27 ligand
CD70 is only expressed on lymphocytes and subsets of dendritic cells upon activation ${ }^{62}$. However, permanent CD70 expression on LSCs allows cell-autonomous CD27 signaling and expansion of LSCs $^{63}$. Similarly, LIGHT is expressed mainly on immune cells upon activation ${ }^{64,65}$. Here, we show that HSPCs express LIGHT and that its expression is upregulated upon activation. Interestingly, LT $\beta \mathrm{R}$ is also expressed at higher levels on activated HSCs. Our in vitro coculture experiments and competitive BM transplant models using Light $^{-1-}$ and control LSKs indicated that LT $\beta$ R and LIGHT act in cis in HSCs and this interaction is crucial for the regulation of self-renewal. In accordance with our findings in HSCs, soluble LIGHT added to Light $^{-/-}$CML LSCs did not rescue colony formation capacity.

LIGHT interacts with three different receptors (HVEM, LT $\beta$ R, and decoy receptor 3 ), two of which are expressed on HSPCs ${ }^{66}$. HVEM signaling in murine HSPCs has been shown to induce myeloid differentiation in response to treatment with LIGHT in vitro and in vivo ${ }^{66}$. Here we show that signaling via LIGHT/ LT $\beta R$ maintained the pool of HSCs and LSCs. Importantly, the phenotype of Light and Ltbr single KO HSCs was similar to Light/ Ltbr double KO HSCs after treatment with 5-FU, excluding a major contribution of other receptors in the maintenance of quiescence. As many other members of the TNF superfamily (e.g., TNF- $\alpha$, Fas-L), LIGHT can act in a soluble or transmembrane form. Importantly, soluble and transmembrane isoforms of TNF superfamily members, including LIGHT, cause different effects on various cell types ${ }^{67-69}$. We now document that neither soluble LIGHT protein nor transmembrane LIGHT expressed by another cell, did rescue the phenotype of Light $^{-1-}$ HSCs, indicating that cell-autonomous LIGHT/LT $\beta$ R signaling is responsible for the observed effects.

LIGHT/LT $\beta$ R signaling maintains stemness by reducing cell proliferation and favoring symmetric over asymmetric cell division. We analyzed the cell division pattern in most experiments 
by defining dividing cells and analyzing Numb distribution according to the cleavage furrow. This analysis has been used by many groups before ${ }^{63,70,71}$, but does not allow to precisely define the axis of cell division. Importantly, the inclusion of an a-tubulin staining confirmed an increased $\mathrm{AD}$ in $\mathrm{Ltbr}^{-1-}$ HSPCs, suggesting that LT $\beta R$ signaling regulates the cell division pattern. Many molecular pathways that regulate stemness and differentiation also regulate apoptosis and vice versa ${ }^{72}$. In the present study, we documented a reduced apoptosis rate in $\mathrm{Ltbr}^{-1-} \mathrm{HSCs}$ that further contributes to the initial cell expansion of more differentiated cells.

It has been shown before that an increase of asymmetric cell division leads to differentiation and exhaustion of stem cells ${ }^{12,73,74}$. This is reflected in an increase in colony formation capacity in first platings and in an improved engraftment after transplantation into first recipient mice, a process that is mediated by both HSCs and progenitors ${ }^{32}$. The exhaustion of functional HSCs leads then to a reduced engraftment in second and third re-platings or re-transplantations.

The absence of LT $\beta R$ signaling induced an upregulation of genes involved in cell cycling and proliferation such as Cdk4 and Cdk6. These cyclin-dependent kinases are known to complex with cyclin D1 to regulate G1/S transition and the exit of HSCs from quiescence ${ }^{75,76}$. Accordingly, $\mathrm{Ltbr}^{-1-}$ HSCs showed a positive correlation with the signature of positive regulation of G1-S transition in GSEA analysis. Similarly, knockdown of $L T B R$ in human $\mathrm{CD} 34^{+}$HSPCs increased the expression of genes involved in cell cycling while reducing genes associated with stemness. Functional analysis of HSCs confirmed a reduced proliferation of LIGHT/LT $\beta R$-competent stem cells, especially of the most primitive LT-HSCs. Moreover, we identified a similar role for LT $\beta R$ in the maintenance and expansion of CML stem cells. Ltbr- and Light-knockout in LSCs reduced the number of leukemia HSPCs in the $\mathrm{BM}$ and prolonged survival in a murine CML model. Importantly, mouse and human CML cells overexpress $L T B R$ and LIGHT when compared to normal HSPCs. Knockdown of LTBR in human CML HSPCs resulted in the downregulation of stemness- and Wnt-related genes, such as MSI2, TNIK, and CTNNB1 and a reduced number of LSCs that form colonies in methylcellulose. Since LSCs are resistant to chemotherapy and probably also TKIs, targeting pathways that regulate and maintain LSCs will be crucial to improve the treatment of leukemia ${ }^{77,78}$. Importantly, LT $\beta R$ deficiency did not affect steady-state hematopoiesis but did only regulate HSC quiescence and cell fate in demand-adapted hematopoiesis. Thus, targeting the LIGHT/ LT $\beta R$ pathway may offer a novel strategy to induce differentiation and to eliminate LSCs.

\section{Methods}

Animals. BL/6 and Ly5.1 mice were purchased from Charles River. Ltbr ${ }^{-1-}$ mice were kindly provided by Prof. A. Aguzzi (Institute of Neuropathology, University Hospital of Zurich, Switzerland; Futterer et al. ${ }^{22}$ ) and Light $^{-1-}$ mice by S. Scheu (University Hospital of Düsseldorf, Germany). KO strains are homozygote mutant mice, generated by intragenic deletions ${ }^{22,23}$. Intercrosses were validated by genotyping PCR and subsequently used for animal husbandry. Light ${ }^{-1-}$ mice on Ly5.1 background were referred to as Ly5.1/Light ${ }^{-1-}$ mice. Offspring mice from Ly5.1 $\times \mathrm{BL} / 6$ breeding are used as recipient mice (referred to as Ly5.1/Ly5.2). C57BL/6-Tg(UBC-GFP)30Scha/J mice were kindly provided by Dr. Mario Bonalli (LASC, University of Zurich, Switzerland) ${ }^{79}$. Mice aged 6-13 weeks were used for experiments. Animal experiments were approved by the local experimental animal committee of the Canton of Bern and performed according to Swiss laws for animal protection.

BM and blood samples from patients. BM samples from untreated staging negative lymphoma patients, who were considered normal by a surgical pathologist and a hematologist, were used as control samples. HSPCs were isolated from apheresis samples of patients that have been treated with chemotherapy and GCSF. PB samples and BM aspirates from untreated CML patients were taken at diagnosis. Patient samples were collected at the University Hospital of Bern after written informed consent. Patient characteristics are listed in Supplementary Tables 1-3. Analysis of samples was approved by the local ethical committee of the Canton of Bern (KEK122/14).

Isolation of BM cells and lineage depletion. Mice were sacrificed; femurs, tibiae, humeri, and the spine were crushed in phosphate-buffered saline (PBS). The BM suspension was filtered through a $40-\mu \mathrm{m}$ cell strainer. Subsequently, red blood cell lysis was performed and cells were washed twice with PBS. Lineage depletion was performed with MACS separation according to the manufacturer's protocol. Briefly, BM cells were stained with biotinylated antibodies (all BioLegend, San Diego, USA) against red cell precursors ( $\alpha$ Ter119), B cells ( $\alpha$ CD19), T cells $(\alpha \mathrm{CD} 3 \varepsilon)$, myeloid cells ( $\alpha \mathrm{Gr} 1)$, and $\alpha$-biotin MicroBeads using LS columns (Miltenyi Biotec, Bergisch Gladbach, Germany).

Isolation of BM niche cells. BM MSCs, ECs, and osteoblasts were isolated according to Schepers et al..$^{80}$. Briefly, bones were cleaned, crushed, and digested with collagenase and DNase. Isolated cells were stained with aCD31-FITC (clone:390) aCD51-APC (clone:RMV-7) aLy-6A/E-PerCP-Cy5.5 (clone:D7, all BioLegend, San Diego, USA) and Streptavidin-V500 (BD Bioscience, Eysins, Switzerland) and hematopoietic cells were excluded using anti-lineage antibodies ( $\alpha$ Ter119, $\alpha \mathrm{CD} 19, \alpha \mathrm{CD} 3 \varepsilon$ and $\alpha \mathrm{Gr} 1, \alpha \mathrm{CD} 45$ (clone:30F11)). BM niche cells were characterized using the following surface markers: EC, $\mathrm{CD} 45^{-} \mathrm{CD} 31^{+} \mathrm{scal}^{+}$; MSC, CD $45^{-} \mathrm{Lin}^{-} \mathrm{CD} 31^{-} \mathrm{sca}^{+}{ }^{-\mathrm{CD}} 51^{+}$; and osteoblasts, $\mathrm{CD} 45^{-} \mathrm{Lin}^{-} \mathrm{CD} 31^{-} \mathrm{sca}^{-} \mathrm{CD} 51^{+}$.

Homing assay. Ly5.1 and $L t b r^{-/-}$LSKs were FACS purified and 26,000 to 54,000 LSKs were injected at a ratio of 1:1 into lethally irradiated Ly5.1/Ly5.2 recipients. Thirteen hours post transplantation the frequency of LSKs homed to the BM cells was analyzed by FACS. The frequency of $\mathrm{Lin}^{-} \mathrm{CD} 45.1^{+}$and $\mathrm{Lin}^{-} \mathrm{CD} 45.2^{+}$cells was calculated as the percentage of transplanted LSKs.

Treatment with 5-FU. BL/6, Ly5.1, and $\mathrm{Ltbr}^{-1-}$ mice were injected intraperitoneally with $150 \mathrm{mg} / \mathrm{kg} 5$-FU (Sigma). Eight to 9 days postinjection, BM cells from Ly5.1 and $L t b r^{-1-}$ mice were analyzed or injected at a ratio of $1: 1$ into lethally irradiated Ly5.1/Ly5.2 recipients $(2 \times 6.5$ Gy with 4 -h interval, Gammacell 40 exactor, Best Theratronics, Ottawa, Canada).

Generation of chimeric mice. A total of 15,000-20,000 LSKs from Ly5.1, $\mathrm{Ltbr}^{-1-}$ or Light ${ }^{-I-}$ mice were injected intravenously at a ratio of 1:1 into lethally irradiated Ly5.1/Ly5.2 or Ly5.1/Light ${ }^{-/-}$recipient mice. Serial re-transplantation experiments were performed $12-18$ weeks after primary transplantation. Ly5.1 and $L t b r^{-1-}$ LSKs from chimeras were FACS purified and injected intravenously (i.v.) at a ratio of 1:1 into lethally irradiated Ly5.1/Ly5.2 recipient mice. If re-transplantation was done with $<5000$ LSKs (third, fourth transplantation) rescue BM cells were given.

CML mouse model. CML was induced in mice ${ }^{45}$. Briefly, FACS-purified LSKs were plated in RPMI medium, supplemented with $10 \%$ fetal calf serum (FCS), $1 \%$ glutamine, $1 \%$ penicillin/streptomycin, $20 \mathrm{ng} / \mathrm{ml}$ thrombopoietin, $100 \mathrm{ng} / \mathrm{ml} \mathrm{SCF}$ overnight, and subsequently transduced by spin infection with BCR-ABL1-GFP retrovirus. CML was induced by i.v. injection of 30,000 transduced LSKs into nonirradiated recipient mice (BL/6 in BL/6: BL/6 CML; $\mathrm{Ltbr}^{-1-}$ in BL/6: $\mathrm{Ltbr}^{-1-}$ CML; Light $^{-1-}$ in BL/6: Light $^{-1-}$ CML). Blood counts were analyzed using a Vet abc Animal Blood Counter (Medical Solution GmbH, Bassersdorf, Switzerland). Mice were analyzed at days 18-20 after CML induction. For secondary transplantations, $5 \times 10^{6} \mathrm{BM}$ cells or 20,000 FACS-purified LSCs from primary CML mice (18-20 days after primary transplantation) were injected i.v. into secondary immunocompetent recipients.

Cell cycle analysis. Ki67 staining was performed with Foxp3/Transcription Factor Staining Buffer Set (eBioscience, San Diego, USA) according to the manufacturer's protocol. In addition, cells were stained with DAPI (Merck, Darmstadt, Germany) and analyzed by FACS. BrdU incorporation was determined according to the manufacturer's protocol (BD Pharmingen BrdU Flow Kits, San Jose, USA).

ImageStream analysis. Numb/a-tubulin (Numb: ab4147, Tubulin: ab7291, Abcam, Cambridge, UK) staining in FACS-purified LSKs or LT/ST-HSCs from chimeric mice, 5-FU-treated BL/6 and $\mathrm{Ltbr}^{-/-}$mice (8 days after treatment) or CML mice was performed as follows: cells were fixed with $4 \%$ paraformaldehyde, followed by permeabilization with $1 \times$ wash buffer (Dako wash, Agilent Technologies, California, USA) and blocking with $10 \%$ normal goat serum (Invitrogen, California, USA) in Dako wash. After overnight incubation at $4{ }^{\circ} \mathrm{C}$ with the primary rabbit $\alpha-N u m b$ antibody or tubulin in Dako diluent, cells were incubated with the secondary antibody (donkey-anti-goat, ab175474; goat-anti-mouse, ab150115) for $1 \mathrm{~h}$ at room temperature ${ }^{63}$. DAPI was used to stain for DNA. Asymmetric cell division was determined by an increase in Numb intensity of 1.8-fold in one of the daughter cells ${ }^{11}$. Cells were acquired using an ImageStreamX MkII imaging flow cytometer (Merck, Darmstadt, Germany). Cells were analyzed using INSPIRE and IDEAS Software ${ }^{63}$. 
Colony-forming cell assays. FACS-purified BM LSKs $\left(600-1 \times 10^{3}\right.$ naive LSKs; $1 \times$ $10^{4}$ 5-FU-treated $\mathrm{Lin}^{-} \mathrm{scal}^{+}$) were plated in MethoCult (STEMCELL Technologies, Cambridge, USA) supplemented with $15 \%$ FCS, $20 \%$ BIT ( $50 \mathrm{mg} / \mathrm{ml}$ bovine serum albumin in Iscove's modified Dulbecco's medium), $1.44 \mathrm{U} / \mathrm{ml}$ rh-insulin and $250 \mathrm{ng} / \mathrm{ml}$ human holo-transferrin, $100 \mu \mathrm{M} 2$-mercaptoethanol, $100 \mathrm{U} / \mathrm{ml}$ penicillin, $100 \mu \mathrm{g} / \mathrm{ml}$ streptomycin, $2 \mathrm{mM}$ L-glutamine, $50 \mathrm{ng} / \mathrm{ml} \mathrm{SCF}$ (rmSCF-1), $10 \mathrm{ng} / \mathrm{ml}$ interleukin-3 (rmIL-3), $10 \mathrm{ng} / \mathrm{ml}$ interleukin-6 (rhIL-6), and $50 \mathrm{ng} / \mathrm{ml}$ fms-related tyrosine kinase 3 ligand (rmFLTL-3). Re-plating was performed with 10,000 cells per dish. For CFU assays of human HSPCs, we plated $1000 \mathrm{CD} 45^{\text {int }} \mathrm{Lin}^{-} \mathrm{CD} 34^{+} \mathrm{BM}$ cells into methylcellulose. Colonies were enumerated after 7-14 days in culture ( $\geq 30$ cells/colony) on a DMIL inverted microscope (Leica, Wetzlar, Germany)

Flow cytometry and cell sorting. Cells were stained in PBS with 5\% FCS with the following antibodies for $30 \mathrm{~min}$ at $4{ }^{\circ} \mathrm{C}$ : aCD117-APC-Cy7 (clone:2B8), aCD48PE-Cy7 (clone:HM48-1), aCD150-APC (clone:TC1512F12.2), aLy-6A/E-PerCPCy5.5, -APC (clone:D7, eBioscience, San Diego, USA), aCD16/CD32-PE-Cy7 (clone:93), aCD34-eFluor-450 (clone:RAM34, eBioscience, San Diego, USA), aCD135-PE, -biotin (clone:A2F10, Novus, Littleton, USA), aCD127-FITC (clone: A7R34), aCD45.1-PerCP-Cy5.5 (clone:A20), aLTßR-PE (clone:ebio3C8, eBioscience, San Diego, USA), aLy-6C-PerCP-Cy5.5 (clone:HK1.4), aCD11b-PECy7, aCD8a-FITC, -APC, FITC (clone:53-6.7), aCD4 (clone:GK1.5), aCD90.1APC (clone:OX-7), aCD90.2-APC (clone:30-H12), Annexin-V-Pacific-Blue, -Alexa Fluor 647, PE, aLy-6G-Pacific-Blue, aCD45.2-Alexa Fluor 700 (clone:104), Ki67PE (clone:16A8), rat IgG2a,k-PE (clone:RTK2758), aCD45-PerCP-Cy5.5, -PE-Cy7, -APC (clone:30F11). Human HSPCs were stained with aCD90-PeCP-Cy5.5 (clone:5E10), aCD34-APC (clone:561), aLT $\beta$ R-PE (clone:31G4D8), $\alpha \mathrm{CD} 38^{-} \mathrm{APC}$ (clone:HIT2), mouse-IgG2b,k (clone:MPC-11), aCD2-biotin (clone:RPA2.10), aCD3E-biotin (clone:OKT3), aCD14-biotin (clone:HCD14), aCD16-biotin (clone:3G8), aCD19-biotin (clone:HIB19), aCD56-biotin (clone:HCD56), aCD235abiotin (clone:HIR2), aCD45-Pacific-Blue (clone:2D1), Streptavidin-V500 (all from BioLegend, San Diego, USA). For intracellular staining of NF- $\mathrm{kB}$ members, cells were surface stained, followed by $4 \%$ paraformaldehyde fixation and permeabilization via ice-cold methanol, according to the manufacturer's protocol. Following primary antibodies were used: phospho-IкBa (Ser32) (clone:14D4), IкBa (clone:44D4); secondary antibody: anti-rabbit IgG $(\mathrm{H}+\mathrm{L}), \mathrm{F}\left(\mathrm{ab}^{\prime}\right) 2$ FragmentAlexa Fluor 647 (all from Cell Signaling Technology Inc., Massachusetts, USA). FACS was performed on a LSR Fortessa cell analyzer or LSRII Flow cytometer (both BD Bioscience, San Jose, USA). Cell sorting was done using a BD FACSARIA III (BD Bioscience, San Jose, USA). Data analysis was performed with Kaluza Flow analysis software (Beckman Coulter, Krefeld, Germany) or FlowJo software (Treestar, Oregon, USA).

RNA isolation and quantitative RT-PCR. RNA was isolated according to the manufacturer's protocol (NucleoSpin RNA XS, Macherey-Nagel, PA, USA) and the expression of genes was analyzed using SYBR Green $2 \times$ PCR Master Mix (Roche, NY, USA) on a 7500 real-time PCR System (AB Biosystems, CA, USA). Actin or Gapdh genes were used for normalization of the gene expression. The sequences of primers are listed in Supplementary Table 4.

\section{High-throughput transcriptome sequencing (RNA-seq). Total RNA was} extracted from FACS-purified $\mathrm{Ltbr}^{-1-}$ and Ly5.1 HSCs ( $\mathrm{Lin}^{-}$Annexin- $\mathrm{V}^{-} \mathrm{scal}^{+}{ }^{+} \mathrm{c}-$ $\mathrm{kit}^{+} \mathrm{CD} 150^{+} \mathrm{CD} 48^{-}$) from chimeric mice using the RNeasy Micro Kit (Qiagen AG, Switzerland) according to the manufacturer's instructions. Total RNA was quality checked on the Bioanalyzer instrument (Agilent Technologies, USA) using the RNA 6000 Pico Chip (Agilent, USA) and quantified by Fluorometry using the QuantiFluor RNA System (Promega, USA).

Library preparation (average library size: $313 \pm 3 \mathrm{bp}$ ) was performed from $20 \mathrm{ng}$ total RNA using the TruSeq Stranded mRNA Library Prep Kit High Throughput (Cat\# RS-122-2103, Illumina, San Diego, CA, USA) and quality (average concentration: $6.7 \pm 1.8 \mathrm{nmol} / \mathrm{L}$ ) was analyzed on the Fragment Analyzer (Advanced Analytical, Ames, IA, USA, High Sensitivity NGS Fragment Analysis Kit, Cat\# DNF-473, Advanced Analytical). Samples were pooled, quantified, adjusted to equal molarity (1.4 pM, QuantiFluor ONE dsDNA System, Cat\# E4871, Promega, Madison, WI, USA), and used for clustering on the NextSeq 500 instrument (Illumina). Samples were sequenced in single reads with 76 bases using the NextSeq 500 High Output Kit 75 cycles (Illumina, Cat\# FC-404-1005). Primary data analysis was performed with the Illumina RTA version 2.4.11 and Basecalling Version bcl2fastq-2.20.0.422. An average per sample of $62 \pm 3.6$ million reads was obtained.

RNA-seq data analysis to access differentially expressed genes. The RNA-seq data were assembled by SeqMan NGen software v.16 and analyzed using ArrayStar software v.16 (DNASTAR, USA). The level of gene expression was assessed using regularized logarithm values from Bioconductor. After statistical analysis, genes with a significant difference in their expression at false discovery rate $p<0.05$ and fold differences $\geq 1.5$ were selected. Data were clustered using standard Euclidean's method based on the average linkage and heatmaps were generated according to the standard normal distribution of the values (Supplementary Dataset 1).
Gene set enrichment analysis. GSEA was performed using GSEA software v.3.0 (Broad Institute). Enrichment analysis was assessed for all pathway-related genes acquired from PathCards database (pathcards.genecards.org).

Transfection of human HSPCs with siRNA. FACS-purified BM CD $34^{+}$HSPCs from patients undergoing a diagnostic $\mathrm{BM}$ aspirate that was considered normal by a hematologist and an independent surgical pathologist (control donor) and CD $34^{+}$HPSCs from the blood of G-CSF-treated patients were cultured in StemSpan SFEM medium in combination with StemSpan CC100 (STEMCELL Technologies) and transfected with control siRNA (siCTRL), LTbR targeting siRNA (siLTBR), or LIGHT targeting siRNA (siLIGHT) (Santa Cruz Biotechnology, Heidelberg, Germany) using TransIT-X2 (Mirus Bio Muttenz, Switzerland) according to the manufacturer's protocol. Genes were analyzed $24 \mathrm{~h}$ post transfection unless otherwise stated.

Statistical analysis. Statistics were calculated using Prism 8.0 (Graph Prism Software, USA). Data were analyzed using one-way analysis of variance and Tukey's or Dunnett's multiple comparison test or Student's $t$ test. Survival curves were analyzed using a log-rank (Mantel-Cox) test. Data are displayed as mean \pm SEM. ${ }^{*} P<0.05,{ }^{* *} P<0.01,{ }^{* * *} P<0.001$, and ${ }^{* * * *} P<0.0001$.

Reporting summary. Further information on research design is available in the Nature Research Reporting Summary linked to this article.

\section{Data availability}

All relevant data are available upon request from the authors. All transcriptomic data compiled for this study have been deposited in NCBI GEO under the accession code: GSE141206. Expression data were derived from a public repository for microarray data (GEO) and are available under accession number GSE32719 (ref. ${ }^{46}$ ) and GSE11675 (ref. ${ }^{47}$ ).

Received: 3 December 2018; Accepted: 17 January 2021; Published online: 16 February 2021

\section{References}

1. Wilson, A. et al. Dormant and self-renewing hematopoietic stem cells and their niches. Ann. NY Acad. Sci. 1106, 64-75 (2007).

2. Will, B. et al. Satb1 regulates the self-renewal of hematopoietic stem cells by promoting quiescence and repressing differentiation commitment. Nat. Immunol. 14, 437-445 (2013).

3. Steinman, R. A. Cell cycle regulators and hematopoiesis. Oncogene 21, 3403-3413 (2002).

4. Fox, R. G., Park, F. D., Koechlein, C. S., Kritzik, M. \& Reya, T. Musashi signaling in stem cells and cancer. Annu. Rev. Cell Dev. Biol. 31, 249-267 (2015).

5. Morrison, S. J. \& Scadden, D. T. The bone marrow niche for haematopoietic stem cells. Nature 505, 327-334 (2014).

6. Riether, C., Schurch, C. M. \& Ochsenbein, A. F. Regulation of hematopoietic and leukemic stem cells by the immune system. Cell Death Differ. 22, 187-198 (2015).

7. Wilson, A. \& Trumpp, A. Bone-marrow haematopoietic-stem-cell niches. Nat. Rev. Immunol. 6, 93-106 (2006).

8. Takizawa, H., Regoes, R. R., Boddupalli, C. S., Bonhoeffer, S. \& Manz, M. G. Dynamic variation in cycling of hematopoietic stem cells in steady state and inflammation. J. Exp. Med. 208, 273-284 (2011).

9. Trumpp, A., Essers, M. \& Wilson, A. Awakening dormant haematopoietic stem cells. Nat. Rev. Immunol. 10, 201-209 (2010).

10. Takizawa, H., Boettcher, S. \& Manz, M. G. Demand-adapted regulation of early hematopoiesis in infection and inflammation. Blood 119, 2991-3002 (2012).

11. Zimdahl, B. et al. Lis1 regulates asymmetric division in hematopoietic stem cells and in leukemia. Nat. Genet. 46, 245-252 (2014).

12. Morrison, S. J. \& Kimble, J. Asymmetric and symmetric stem-cell divisions in development and cancer. Nature 441, 1068-1074 (2006).

13. Deshpande, A. J., Ahmed, F. \& Buske, C. Identification of murine and human acute myeloid leukemia stem cells. Methods Mol. Biol. 568, 21-35 (2009).

14. Reya, T. et al. A role for Wnt signalling in self-renewal of haematopoietic stem cells. Nature 423, 409-414 (2003).

15. Sugiyama, T., Kohara, H., Noda, M. \& Nagasawa, T. Maintenance of the hematopoietic stem cell pool by CXCL12-CXCR4 chemokine signaling in bone marrow stromal cell niches. Immunity 25, 977-988 (2006).

16. Wiesmann, A. et al. Expression of CD27 on murine hematopoietic stem and progenitor cells. Immunity 12, 193-199 (2000). 
17. Nolte, M. A. et al. Immune activation modulates hematopoiesis through interactions between CD27 and CD70. Nat. Immunol. 6, 412-418 (2005).

18. Schurch, C., Riether, C., Matter, M. S., Tzankov, A. \& Ochsenbein, A. F. CD27 signaling on chronic myelogenous leukemia stem cells activates Wnt target genes and promotes disease progression. J. Clin. Invest. 122, 624-638 (2012).

19. Riether, C. et al. Tyrosine kinase inhibitor-induced CD70 expression mediates drug resistance in leukemia stem cells by activating Wnt signaling. Sci. Transl. Med. 7, 298ra119 (2015).

20. Gommerman, J. L., Browning, J. L. \& Ware, C. F. The lymphotoxin network: orchestrating a type I interferon response to optimize adaptive immunity. Cytokine Growth Factor Rev. 25, 139-145 (2014).

21. Schneider, K., Potter, K. G. \& Ware, C. F. Lymphotoxin and LIGHT signaling pathways and target genes. Immunol. Rev. 202, 49-66 (2004)

22. Futterer, A., Mink, K., Luz, A., Kosco-Vilbois, M. H. \& Pfeffer, K. The lymphotoxin beta receptor controls organogenesis and affinity maturation in peripheral lymphoid tissues. Immunity 9, 59-70 (1998).

23. Scheu, S. et al. Targeted disruption of LIGHT causes defects in costimulatory $\mathrm{T}$ cell activation and reveals cooperation with lymphotoxin beta in mesenteric lymph node genesis. J. Exp. Med. 195, 1613-1624 (2002).

24. Madge, L. A., Kluger, M. S., Orange, J. S. \& May, M. J. Lymphotoxin-alpha 1 beta 2 and LIGHT induce classical and noncanonical NF-kappa B-dependent proinflammatory gene expression in vascular endothelial cells. J. Immunol. 180, 3467-3477 (2008)

25. Ehlers, S. et al. The lymphotoxin beta receptor is critically involved in controlling infections with the intracellular pathogens Mycobacterium tuberculosis and Listeria monocytogenes. J. Immunol. 170, 5210-5218 (2003).

26. Wege, A. K., Huber, B., Wimmer, N., Mannel, D. N. \& Hehlgans, T. LTbetaR expression on hematopoietic cells regulates acute inflammation and influences maturation of myeloid subpopulations. Innate Immun. 20, 461-470 (2013).

27. Lukashev, M. et al. Targeting the lymphotoxin-beta receptor with agonist antibodies as a potential cancer therapy. Cancer Res. 66, 9617-9624 (2006).

28. Bajaj, J., Zimdahl, B. \& Reya, T. Fearful symmetry: subversion of asymmetric division in cancer development and progression. Cancer Res. 75, 792-797 (2015).

29. Kondo, M., Weissman, I. L. \& Akashi, K. Identification of clonogenic common lymphoid progenitors in mouse bone marrow. Cell 91, 661-672 (1997).

30. Akashi, K., Traver, D., Miyamoto, T. \& Weissman, I. L. A clonogenic common myeloid progenitor that gives rise to all myeloid lineages. Nature 404, 193-197 (2000).

31. Eaves, C. J. Hematopoietic stem cells: concepts, definitions, and the new reality. Blood 125, 2605-2613 (2015).

32. Coulombel, L. Identification of hematopoietic stem/progenitor cells: strength and drawbacks of functional assays. Oncogene 23, 7210-7222 (2004).

33. Humphries, R. K., Eaves, A. C. \& Eaves, C. J. Self-renewal of hemopoietic stem cells during mixed colony formation in vitro. Proc. Natl Acad. Sci. USA 78, 3629-3633 (1981).

34. Daller, B. et al. Lymphotoxin-beta receptor activation by lymphotoxin-alpha (1)beta(2) and LIGHT promotes tumor growth in an NFkappaB-dependent manner. Int. J. Cancer 128, 1363-1370 (2011).

35. Chen, M. C. et al. The role of apoptosis signal-regulating kinase 1 in lymphotoxin-beta receptor-mediated cell death. J. Biol. Chem. 278, 16073-16081 (2003).

36. Eissner, G., Kolch, W. \& Scheurich, P. Ligands working as receptors: reverse signaling by members of the TNF superfamily enhance the plasticity of the immune system. Cytokine Growth Factor Rev. 15, 353-366 (2004).

37. Lim, S. G., Suk, K. \& Lee, W. H. Reverse signaling from LIGHT promotes proinflammatory responses in the human monocytic leukemia cell line, $\mathrm{THP}^{-} 1$. Cell Immunol. 285, 10-17 (2013).

38. Pawliuk, R., Eaves, C. \& Humphries, R. K. Evidence of both ontogeny and transplant dose-regulated expansion of hematopoietic stem cells in vivo. Blood 88, 2852-2858 (1996).

39. Iscove, N. N. \& Nawa, K. Hematopoietic stem cells expand during serial transplantation in vivo without apparent exhaustion. Curr. Biol. 7, 805-808 (1997).

40. $\mathrm{Wu}, \mathrm{M}$. et al. Imaging hematopoietic precursor division in real time. Cell Stem Cell 1, 541-554 (2007).

41. Guo, M., Jan, L. Y. \& Jan, Y. N. Control of daughter cell fates during asymmetric division: interaction of Numb and Notch. Neuron 17, 27-41 (1996).

42. Harrison, D. E. \& Lerner, C. P. Most primitive hematopoietic stem-cells are stimulated to cycle rapidly after treatment with 5-fluorouracil. Blood 78, 1237-1240 (1991).

43. Randall, T. D. \& Weissman, I. L. Phenotypic and functional changes induced at the clonal level in hematopoietic stem cells after 5-fluorouracil treatment. Blood 89, 3596-3606 (1997).

44. Liu, T., Zhang, L., Joo, D. \& Sun, S. C. NF-kappaB signaling in inflammation. Signal Transduct. Target Ther. 2, 17023 (2017).

45. Mumprecht, S. et al. Defective homing and impaired induction of cytotoxic T cells by BCR/ABL-expressing dendritic cells. Blood 113, 4681-4689 (2009).
46. Pang, W. W. et al. Human bone marrow hematopoietic stem cells are increased in frequency and myeloid-biased with age. Proc. Natl Acad. Sci. USA 108, 20012-20017 (2011)

47. Lemoli, R. M. et al. Molecular and functional analysis of the stem cell compartment of chronic myelogenous leukemia reveals the presence of a CD34- cell population with intrinsic resistance to imatinib. Blood 114, 5191-5200 (2009)

48. Zhao, J. L. \& Baltimore, D. Regulation of stress-induced hematopoiesis. Curr Opin. Hematol. 22, 286-292 (2015).

49. Schuettpelz, L. G. \& Link, D. C. Regulation of hematopoietic stem cell activity by inflammation. Front. Immunol. 4, 204 (2013).

50. Zhao, J. L. et al. Conversion of danger signals into cytokine signals by hematopoietic stem and progenitor cells for regulation of stress-induced hematopoiesis. Cell Stem Cell 14, 445-459 (2014).

51. Orford, K. W. \& Scadden, D. T. Deconstructing stem cell self-renewal: genetic insights into cell-cycle regulation. Nat. Rev. Genet. 9, 115-128 (2008).

52. Rossi, L. et al. Less is more: unveiling the functional core of hematopoietic stem cells through knockout mice. Cell Stem Cell 11, 302-317 (2012).

53. Pietras, E. M., Warr, M. R. \& Passegue, E. Cell cycle regulation in hematopoietic stem cells. J. Cell Biol. 195, 709-720 (2011).

54. Hill, R. \& Wu, H. PTEN, stem cells, and cancer stem cells. J. Biol. Chem. 284, 11755-11759 (2009)

55. Reya, T. \& Clevers, H. Wnt signalling in stem cells and cancer. Nature 434, 843-850 (2005)

56. Rebel, V. I. et al. Essential role for the p55 tumor necrosis factor receptor in regulating hematopoiesis at a stem cell level. J. Exp. Med. 190, 1493-1504 (1999).

57. Solanilla, A. et al. CD40-ligand stimulates myelopoiesis by regulating flt3ligand and thrombopoietin production in bone marrow stromal cells. Blood 95, 3758-3764 (2000).

58. Kim, Y. S., Nedospasov, S. A. \& Liu, Z. G. TRAF2 plays a key, nonredundant role in LIGHT-lymphotoxin beta receptor signaling. Mol. Cell. Biol. 25, 2130-2137 (2005).

59. Ware, C. F. Network communications: lymphotoxins, LIGHT, and TNF Annu. Rev. Immunol. 23, 787-819 (2005).

60. Nakano, H. et al. TRAF5, an activator of NF-kappaB and putative signal transducer for the lymphotoxin-beta receptor. J. Biol. Chem. 271, 14661-14664 (1996)

61. Stein, S. J. \& Baldwin, A. S. Deletion of the NF-kappaB subunit p65/RelA in the hematopoietic compartment leads to defects in hematopoietic stem cell function. Blood 121, 5015-5024 (2013).

62. Tesselaar, K. et al. Expression of the murine CD27 ligand CD70 in vitro and in vivo. J. Immunol. 170, 33-40 (2003).

63. Riether, C. et al. CD70/CD27 signaling promotes blast stemness and is a viable therapeutic target in acute myeloid leukemia. J. Exp. Med. 214, 359-380 (2017).

64. Wang, Y., Zhu, M., Miller, M. \& Fu, Y. X. Immunoregulation by tumor necrosis factor superfamily member LIGHT. Immunol. Rev. 229, 232-243 (2009).

65. Croft, M. The role of TNF superfamily members in T-cell function and diseases. Nat. Rev. Immunol. 9, 271-285 (2009).

66. Chen, W. et al. Hematopoietic stem/progenitor cell differentiation towards myeloid lineage is modulated by LIGHT/LIGHT receptor signaling. J. Cell. Physiol. 233, 1095-1103 (2017).

67. Ardestani, S. et al. Membrane versus soluble isoforms of TNF-alpha exert opposing effects on tumor growth and survival of tumor-associated myeloid cells. Cancer Res. 73, 3938-3950 (2013).

68. LA, O. R. et al. Membrane-bound Fas ligand only is essential for Fas-induced apoptosis. Nature 461, 659-663 (2009).

69. del Rio, M. L., Schneider, P., Fernandez-Renedo, C., Perez-Simon, J. A. \& Rodriguez-Barbosa, J. I. LIGHT/HVEM/LTbetaR interaction as a target for the modulation of the allogeneic immune response in transplantation. Am. J. Transplant. 13, 541-551 (2013)

70. Tominaga, K. et al. Semaphorin signaling via MICAL3 induces symmetric cell division to expand breast cancer stem-like cells. Proc. Natl Acad. Sci. USA 116, 625-630 (2019).

71. Hinge, A. et al. p190-B RhoGAP and intracellular cytokine signals balance hematopoietic stem and progenitor cell self-renewal and differentiation. Nat. Commun. 8, 14382 (2017)

72. Alenzi, F. Q. et al. The haemopoietic stem cell: between apoptosis and self renewal. Yale J. Biol. Med. 82, 7-18 (2009).

73. Viatour, P. Bridges between cell cycle regulation and self-renewal maintenance. Genes Cancer 3, 670-677 (2012).

74. Wilson, A. et al. Hematopoietic stem cells reversibly switch from dormancy to self-renewal during homeostasis and repair. Cell 135, 1118-1129 (2008).

75. Kozar, K. et al. Mouse development and cell proliferation in the absence of Dcyclins. Cell 118, 477-491 (2004).

76. Malumbres, M. et al. Mammalian cells cycle without the D-type cyclindependent kinases Cdk4 and Cdk6. Cell 118, 493-504 (2004).

77. Holyoake, T. L. \& Vetrie, D. The chronic myeloid leukemia stem cell: stemming the tide of persistence. Blood 129, 1595-1606 (2017). 
78. Essers, M. A. \& Trumpp, A. Targeting leukemic stem cells by breaking their dormancy. Mol. Oncol. 4, 443-450 (2010).

79. Schaefer, B. C., Schaefer, M. L., Kappler, J. W., Marrack, P. \& Kedl, R. M. Observation of antigen-dependent CD8+ T-cell/ dendritic cell interactions in vivo. Cell Immunol. 214, 110-122 (2001).

80. Schepers, K. et al. Myeloproliferative neoplasia remodels the endosteal bone marrow niche into a self-reinforcing leukemic niche. Cell Stem Cell 13 , 285-299 (2013).

\section{Acknowledgements}

Data analyzed in this paper were generated in collaboration with the Genetic Diversity Centre (GDC, ETH Zürich, Zurich, Switzerland) and the Flow cytometry facility (FACSlab, University Bern, Bern, Switzerland). This work is supported by the Swiss National Science Foundation, Swiss Cancer League, Bernische Krebsliga, and the Stiftung zur Krebsbekämpfung.

\section{Author contributions}

S.S.H. designed, performed, analyzed experiments, interpreted results, and wrote the manuscript. A.R. and R.R. designed, performed experiments, and analyzed data. M.A.A. performed experiments. D.K. performed experiments. C.R. designed and analyzed data and interpreted the results. G.M.B. collected and prepared patient samples, interpreted data, and revised the manuscript. A.F.O. directed the study, analyzed and interpreted results, and wrote the manuscript. All authors revised the manuscript and approved its final version.

\section{Competing interests}

The authors declare no competing interests.

\section{Additional information}

Supplementary information The online version contains supplementary material available at https://doi.org/10.1038/s41467-021-21317-x.

Correspondence and requests for materials should be addressed to A.F.O.

Peer review information Nature Communications thanks Takahiro Ito, Tannishtha Reya and Hitoshi Takizawa for their contribution to the peer review of this work.

Reprints and permission information is available at http://www.nature.com/reprints

Publisher's note Springer Nature remains neutral with regard to jurisdictional claims in published maps and institutional affiliations.

(c) (i) Open Access This article is licensed under a Creative Commons Attribution 4.0 International License, which permits use, sharing, adaptation, distribution and reproduction in any medium or format, as long as you give appropriate credit to the original author(s) and the source, provide a link to the Creative Commons license, and indicate if changes were made. The images or other third party material in this article are included in the article's Creative Commons license, unless indicated otherwise in a credit line to the material. If material is not included in the article's Creative Commons license and your intended use is not permitted by statutory regulation or exceeds the permitted use, you will need to obtain permission directly from the copyright holder. To view a copy of this license, visit http://creativecommons.org/ licenses/by/4.0/.

(c) The Author(s) 2021 